\title{
Chi-boundedness of graph classes excluding wheel vertex-minors
}

\author{
Hojin $\mathrm{Choi}^{1}$, O-joung Kwon ${ }^{* 2}$, Sang-il Oum ${ }^{\dagger 1}$, and Paul Wollan ${ }^{\ddagger 3}$ \\ ${ }^{1}$ Department of Mathematical Sciences, KAIST, Daejeon, South Korea. \\ ${ }^{2}$ Department of Mathematics, Incheon National University, Incheon, South Korea. \\ ${ }^{3}$ Department of Computer Science, University of Rome, "La Sapienza", Rome, Italy.
}

March 15, 2018

\begin{abstract}
A class of graphs is $\chi$-bounded if there exists a function $f: \mathbb{N} \rightarrow \mathbb{N}$ such that for every graph $G$ in the class and an induced subgraph $H$ of $G$, if $H$ has no clique of size $q+1$, then the chromatic number of $H$ is less than or equal to $f(q)$. We denote by $W_{n}$ the wheel graph on $n+1$ vertices. We show that the class of graphs having no vertex-minor isomorphic to $W_{n}$ is $\chi$-bounded. This generalizes several previous results; $\chi$-boundedness for circle graphs, for graphs having no $W_{5}$ vertex-minors, and for graphs having no fan vertex-minors.
\end{abstract}

\section{Introduction}

All graphs in this paper are simple and undirected. A clique of a graph is a set of pairwise adjacent vertices. The clique number of a graph $G$, denoted by $\omega(G)$, is the maximum number of vertices in a clique in $G$. We denote the chromatic number of a graph $G$ by $\chi(G)$.

Gyárfás [15] introduced the concept of a $\chi$-bounded class of graphs. A class $\mathcal{C}$ of graphs is $\chi$-bounded if there exists a function $f: \mathbb{N} \rightarrow \mathbb{N}$ such that for every graph $G \in \mathcal{C}$ and an induced subgraph $H$ of $G, \chi(H) \leqslant f(\omega(H))$. Such a function $f$ is called a $\chi$-bounding function. Gyárfás [15] proved that for every positive integer $k$, the class of graphs with no induced path of length $k$ is $\chi$-bounded.

A vertex-minor of a graph $G$ is an induced subgraph of a graph that can be obtained from $G$ by a sequence of local complementations [1, 2, 3, 4, 5, 17]. The precise definition will be presented in Section 2 .

\footnotetext{
*Previous affiliation : Logic and Semantics, Technische Universität Berlin, Berlin, Germany. Supported by the European Research Council (ERC) under the European Union's Horizon 2020 research and innovation programme (ERC consolidator grant DISTRUCT, agreement No. 648527).

${ }^{\dagger}$ Supported by the National Research Foundation of Korea (NRF) grant funded by the Korea government (MSIT) (No. NRF-2017R1A2B4005020).

${ }^{\ddagger}$ Supported by the European Research Council (ERC) under the European Union’s Seventh Framework Programme (FP7/2007-2013)/ERC Grant Agreement no. 279558.

${ }^{0}$ E-mail addresses: hjchoi0330@gmail.com (H. Choi), ojoungkwon@gmail.com (O. Kwon), sangil@kaist.edu (S. Oum), paul.wollan@gmail.com (P. Wollan)
} 
As graph minors are motivated by the study of planar graphs, one of the major motivations to study vertex-minors is due to its close relation to circle graphs. Circle graphs are intersection graphs of chords on a circle. Vertex-minors of a circle graph are circle graphs, as local complementations preserve the property of being circle graphs.

Gyárfás [13, 14] proved the following theorem.

Theorem 1.1 (Gyárfás [13, 14]). The class of circle graphs is $\chi$-bounded.

Dvořák and Král' [10] proved that graphs of rank-width at most $k$ are also $\chi$-bounded and it is also the case that the class of graphs of rank-width at most $k$ is closed under taking vertex-minors.

These motivate the following conjecture of Geelen (see [10]).

Conjecture 1.2 (Geelen (see [10])). For every graph $H$, the class of graphs with no $H$ vertex-minor is $\chi$-bounded.

Conjecture 1.2 is known to be true for the following cases. Here, for an integer $k \geqslant 3$, a wheel graph $W_{k}$ is a graph that consists of an induced cycle on $k$ vertices and an additional vertex adjacent to all vertices of the induced cycle.

(I) Conjecture 1.2 is true if $H$ is a vertex-minor of $W_{5}$, as shown by Dvoráa and Král' [10]. Bouchet [6] proved that a graph is a circle graph if and only if the graph has none of $W_{5}, W_{7}$, and $F_{7}$ as a vertex-minor, where $F_{7}$ is the (unique) 7-vertex bipartite graph such that $F_{7}-v$ is a cycle of length 6 for some vertex $v$ of degree 3. Geelen [11 gave a decomposition theorem of graphs with no $W_{5}$ vertex-minor, using circle graphs as one of the building blocks by applying a theorem of Bouchet. Dvořák and Král' [10 used the decomposition theorem of Geelen and Theorem 1.1 to prove that the class of graphs with no $W_{5}$ vertex-minor is $\chi$-bounded.

(II) Conjecture 1.2 is true if $H$ is a vertex-minor of a fan graph (a fan graph is a graph obtained from the wheel graph by removing a vertex of degree 3), as shown by I. Choi, Kwon, and Oum [7].

This implies that Conjecture 1.2 is true for all $H$ such that $H$ is a cycle, as every cycle is a vertex-minor of a sufficiently large fan graph. For such $H$, the conjecture also follows from a recent theorem of Chudnovsky, Seymour, and Scott [8], proving a conjecture of Gyárfás that the class of graphs having no induced cycles of length at least $k$ is $\chi$-bounded for all $k$.

We prove Conjecture 1.2 for $H=W_{k}$ for all $k \geqslant 3$, thus implying both (I) and (II).

Theorem 8.2. For every integer $n \geqslant 3$, the class of graphs with no $W_{n}$ vertex-minor is $\chi$-bounded.

Our theorem also provides an alternative proof of Theorem 1.1, as $W_{n}$ is not a circle graph for $n \geqslant 5$. Of course, (I) implies Theorem [1.1, but the proof of (I) by Dvořák and Král' [10] depends on Theorem 1.1. Moreover, (II) does not imply Theorem 1.1 since a fan graph is a circle graph.

The paper is organized as follows. Section 2 provides some preliminary concepts. Section 3 gives a high level overview of the proof of our main theorem. Section 4 presents a lemma that will help us to arrange finite sets of reals. Section 5 proves a variant of Ramsey's theorem. In Sections 6 and 7 , we explain how to obtain a wheel graph from several large graphs as a vertex-minor. We prove our main theorem in Section 8 . 

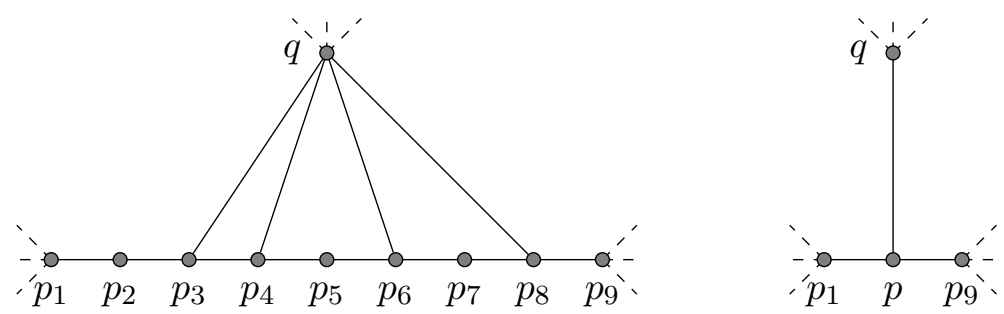

Figure 1: The graph $G$ and a contraction $G /\left\{p_{2}, p_{3}, \ldots, p_{8}\right\}$.

\section{Preliminaries}

For a graph $G$, let $V(G)$ and $E(G)$ denote the vertex set and the edge set of $G$, respectively. Let $G$ be a graph. For $S \subseteq V(G)$, we denote by $G[S]$ the subgraph of $G$ induced by $S$. For $v \in V(G)$ and $S \subseteq V(G)$, we denote by $G-v$ the graph obtained from $G$ by removing $v$, and by $G-S$ the graph obtained by removing all vertices in $S$. For $F \subseteq E(G)$, we denote by $G-F$ the graph with vertex set $V(G)$ and edge set $E(G) \backslash F$. For $v \in V(G)$, the set of neighbors of $v$ in $G$ is denoted by $N_{G}(v)$, and the degree of $v$ is the size of $N_{G}(v)$. For $S \subseteq V(G)$, we denote by $N_{G}(S)$ the set of vertices in $V(G) \backslash S$ having a neighbor in $S$. For an edge $e$ of a graph $G$, we denote by $G / e$ the graph obtained by contracting $e$. Note we are only considering simple graphs, so we delete any parallel edges which arise from contracting an edge. A graph $H$ is a subdivision of $G$ if $H$ can be obtained from $G$ by replacing each edge $v w$ by a path with at least one edge whose end vertices are $v$ and $w$.

For a vertex $v$ in a graph $G$, to perform local complementation at $v$, replace the subgraph of $G$ induced on $N_{G}(v)$ by its complement graph. We write $G * v$ to denote the graph obtained from $G$ by applying local complementation at $v$. Two graphs $G$ and $H$ are locally equivalent if $G$ can be obtained from $H$ by a sequence of local complementations. A graph $H$ is a vertex-minor of a graph $G$ if $H$ is an induced subgraph of a graph which is locally equivalent to $G$.

For an edge $u v$ of a graph $G$, to pivot the edge $u v$ in $G$, denoted $G \wedge u v$, perform the series of local complementations $G * u * v * u$. Note that $G \wedge u v$ is identical to the graph obtained from $G$ by flipping the adjacency relation between every pair of vertices $x$ and $y$ where $x$ and $y$ are contained in distinct sets of $N_{G}(u) \backslash\left(N_{G}(v) \cup\{v\}\right), N_{G}(v) \backslash\left(N_{G}(u) \cup\{v\}\right)$, and $N_{G}(u) \cap N_{G}(v)$, and finally swapping the labels of $u$ and $v$. To flip the adjacency relation between two vertices, we delete the edge if it exists and add it otherwise.

For a vertex $v$ of a graph $G$ with exactly two neighbors $v_{1}$ and $v_{2}$ that are non-adjacent, the series of operations $(G * v)-v$ is called smoothing the vertex $v$. The resulting graph is equivalently the graph obtained by contracting an edge incident with $v$. Note that if $H$ is a subdivision of $G$, then $G$ is a vertex-minor of $H$ because we can construct $G$ from $H$ by repeatedly smoothing vertices.

We describe another type of contraction that creates a graph isomorphic to a vertex-minor of the original graph. For a vertex set $S$ of a graph $G$ where $G[S]$ is connected, we denote by $G / S$ the graph obtained by contracting all edges in $G[S]$. Thus, all vertices in $S$ are identified to one vertex in $G / S$. In general, $G / S$ is not a vertex-minor of $G$; the following lemma describes a situation, which will be useful in the coming arguments, where $G / S$ is isomorphic to a vertex-minor of $G$.

Lemma 2.1. Let $m \geqslant 4$ be an integer. Let $G$ be a graph and let $\left\{p_{1}, \ldots, p_{m}\right\} \cup\{q\}$ be a vertex set of $G$ such that 
- $p_{1} p_{2} \cdots p_{m}$ is an induced path in $G$,

- there are no edges between $\left\{p_{2}, \ldots, p_{m-1}\right\}$ and $V(G) \backslash\left(\left\{p_{1}, \ldots, p_{m}\right\} \cup\{q\}\right)$,

- $q$ has at least one neighbor in $\left\{p_{3}, \ldots, p_{m-1}\right\}$, and no neighbors in $\left\{p_{1}, p_{2}, p_{m}\right\}$.

Then $G /\left\{p_{2}, p_{3}, \ldots, p_{m-1}\right\}$ is isomorphic to a vertex-minor of $G$.

Proof. Let $G^{\prime}:=G /\left\{p_{2}, p_{3}, \ldots, p_{m-1}\right\}$ and let $p$ be the contracted vertex in $G^{\prime}$. We depict in Figure 1. We simulate this contraction as follows:

1. First if there is a vertex of degree 2 in $\left\{p_{3}, \ldots, p_{m-1}\right\}$, then we smooth it. We may assume that there are no vertices of degree 2 in $p_{3}, \ldots, p_{m-1}$.

2. If $m \geqslant 7$, then we apply local complementation at $p_{4}$ and remove it. This local complementation removes edges $q p_{3}$ and $q p_{5}$, and links $p_{3}$ and $p_{5}$. Then we smooth $p_{3}$ and $p_{5}$. By applying this procedure repeatedly, we may assume $m \in\{4,5,6\}$.

3. If $m=4$, then we smooth $p_{2}$. If $m=5$, then we apply local complementation at $p_{3}$ and remove it, and then smooth $p_{4}$. If $m=6$, then we pivot $p_{3} p_{4}$ and remove $p_{3}$ and $p_{4}$, and then smooth $p_{5}$.

It is not difficult to see that each resulting graph is isomorphic to $G^{\prime}$.

\section{Overview of the approach}

We begin by taking a leveling of the given graph. A sequence $L_{0}, L_{1}, \ldots, L_{m}$ of disjoint subsets of the vertex set of a graph $G$ is called a leveling in $G$ if

1. $\left|L_{0}\right|=1$, and

2. for each $i \in\{1, \ldots, m\}$, every vertex in $L_{i}$ has a neighbor in $L_{i-1}$, and has no neighbors in $L_{j}$ for all $j \in\{0, \ldots, i-2\}$.

Each $L_{i}$ is called a level. We can obtain a leveling that covers all vertices in a connected graph by fixing a vertex $v$, and taking $L_{i}$ as the set of all vertices at distance $i$ from $v$. Given a leveling $L_{0}, L_{1}, \ldots, L_{m}$, if each $L_{i}$ can be colored by $x$ colors, then the whole graph can be colored by $2 x$ colors, because there are no edges between $L_{i}$ and $L_{j}$ for $|j-i| \geqslant 2$. Therefore, starting with a connected graph with sufficiently large chromatic number, we may assume that there is some level $L_{i}$ that still has large chromatic number.

A natural approach to find a wheel vertex-minor is to find a long induced cycle in the level $L_{i}$ with large chromatic number, using the result by Chudnovsky, Scott, and Seymour (Theorem 8.1), and then to construct a large wheel vertex-minor using the connected subgraph on $L_{0} \cup \cdots \cup L_{i-1}$. However, this strategy does not work well. For instance, we may find a graph depicted in the first figure of Figure 2. In this graph, if we apply local complementations to create edges from $v$ to the bottom cycle, then we obtain a graph obtained from a large wheel by adding some parallel chords, depicted in the right-hand figure. At this point, it is difficult to remove these chords to finally obtain a wheel graph as a vertex-minor.

To avoid such problems, we aim to find a similar structure, but having two disjoint large independent sets having regular neighbors on the cycle. One simple example is depicted in the first 

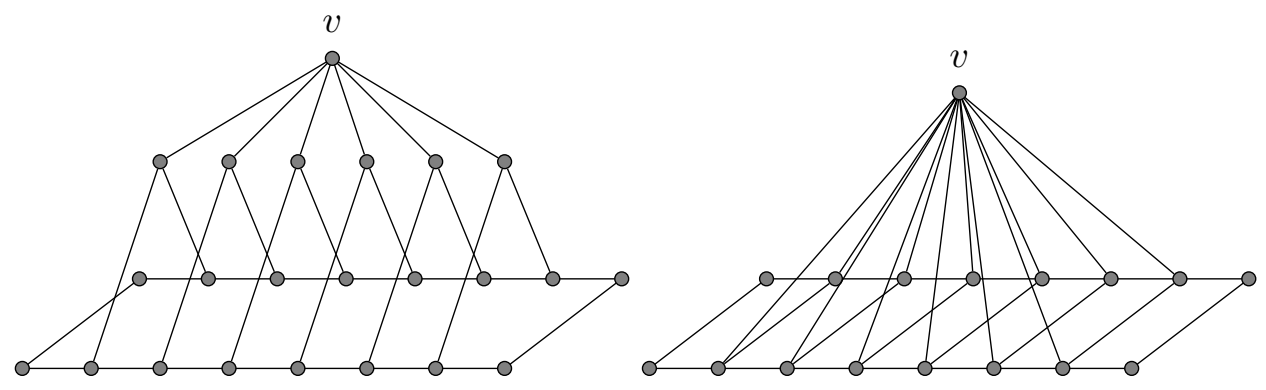

Figure 2: A problematic case.
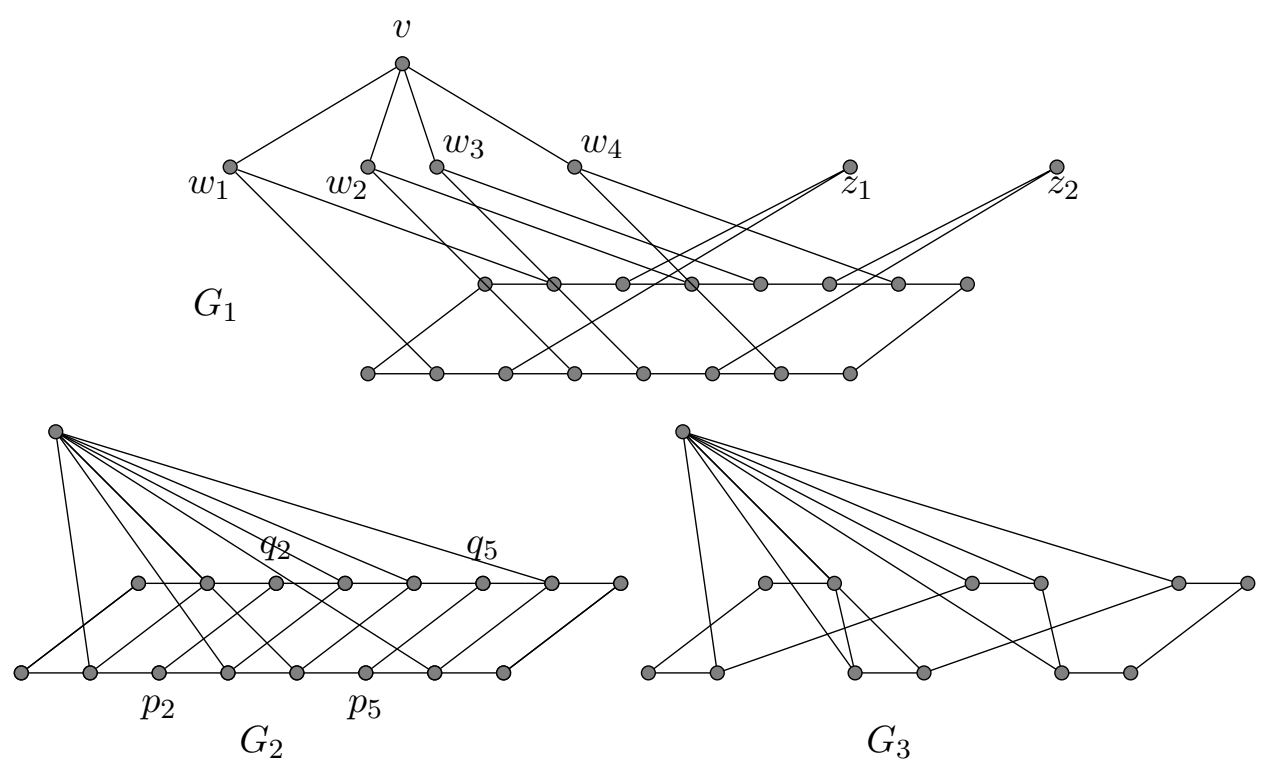

Figure 3: An example procedure to find a large wheel. The graph $G_{2}$ is $\left(G_{1} * w_{1} * w_{2} * w_{3} * w_{4} *\right.$ $\left.z_{1} * z_{2}\right)-\left\{w_{1}, w_{2}, w_{3}, w_{4}, z_{1}, z_{2}\right\}$, and the graph $G_{3}$ is $\left(G_{2} \wedge p_{2} q_{2} \wedge p_{5} q_{5}\right)-\left\{p_{2}, p_{5}, q_{2}, q_{5}\right\}$. Since $G_{3}$ is isomorphic to a subdivision of $W_{8}$, it contains $W_{8}$ as a vertex-minor.

figure of Figure 3. In this example, one independent set of vertices $w_{i}$ is used to create a vertex having many neighbors on the cycle, and the second set of vertices $z_{j}$ is used to remove the newly created chords. We depict this procedure in Figure 3. Briefly speaking, to remove the chords that are newly created from $w_{i}$ 's, we want to add new chords that does not share an end vertex with chords created from $w_{i}$ 's, and then by pivoting these edges, we remove chords created from $w_{i}$ 's. We need more involved arguments for dealing with general cases. This is one of the main procedures we will utilize to find a large wheel as a vertex-minor.

Our argument begins with a structure arising from recursively taking repeated levelings. Explicitly, we aim to find pairwise disjoint vertex sets $X_{i}$ and $Y_{1}, \ldots, Y_{i}$ and $Z_{1}, \ldots, Z_{i}$ in a graph $G$ with sufficiently large $i$ such that

- $G\left[X_{i}\right]$ has large chromatic number,

- for each vertex $v \in X_{i}$ and each $x \in\{1, \ldots, i\}, v$ has a neighbor in $Y_{x}$ and no neighbors in $Z_{x}$, 
- for each $x \in\{1, \ldots, i\}$, every vertex in $Y_{x}$ has a neighbor in $Z_{x}$,

- for each $x \in\{1, \ldots, i\}$, there exists a vertex $r_{x} \in Z_{x}$ where for every $v \in N_{G}\left(Y_{x}\right) \cap Z_{x}$, there is a path $P$ from $v$ to $r_{x}$ in $G\left[Z_{x}\right]$ with $N_{G}\left(Y_{x}\right) \cap V(P)=\{v\}$,

- for distinct integers $x, y \in\{1, \ldots, i\}$ with $x<y$, there are no edges between $Z_{x}$ and $Z_{y} \cup Y_{y}$.

Assume that we have such $X_{i}, Y_{1}, \ldots, Y_{i}, Z_{1}, \ldots, Z_{i}$. If $\chi\left(G\left[X_{i}\right]\right)$ is sufficiently large, then some connected component $C$ of $G\left[X_{i}\right]$ has the same chromatic number as $G\left[X_{i}\right]$. We choose a vertex $v$ in $C$, and we take a leveling $L_{0}, L_{1}, \ldots, L_{m}$ of $C$ where $L_{i}$ is the set of all vertices at distance $i$ from $v$. Then there is a level $L_{t}$ such that $\chi\left(G\left[L_{t}\right]\right) \geqslant \chi\left(G\left[X_{i}\right]\right) / 2$. If $t=1$, we find a long induced cycle in $G\left[L_{1}\right]$ and thus we can obtain a large wheel vertex-minor directly. Otherwise, it holds that $t \geqslant 2$. Assign $X_{i+1}:=L_{t}$ and $Y_{i+1}:=L_{t-1}$ and $Z_{i+1}:=L_{0} \cup L_{1} \cup \cdots \cup L_{t-2}$. Thus, by requiring $\chi\left(G\left[X_{i}\right]\right)$ to be sufficiently large, we can either find $X_{i+1}, Y_{1}, \ldots, Y_{i+1}, Z_{1}, \ldots, Z_{i+1}$ with the desired properties, or find a large wheel vertex-minor.

From this structure, we will reduce to several types of simpler graphs step by step in Sections 6 and 7. We first find a long induced cycle $C=q_{1} q_{2} \cdots q_{m} q_{1}$ in $G\left[X_{i}\right]$ using the result of Chudnovsky, Scott, and Seymour (Theorem 8.1). Secondly, we obtain a structure called a $(w, \ell)$-patched cycle where $w=i$. The definition will be rigorously given in Section 7 , for the moment, we proceed more informally. A $(w, \ell)$-patched cycle consists of $C$ along with vertex sets $S_{j}=\left\{s_{1}^{j}, s_{2}^{j}, \ldots, s_{\ell}^{j}\right\} \subseteq Y_{j}$ for each $j \in\{1,2, \ldots, i\}$ and a sequence of vertices $q_{b_{1}}, q_{b_{2}}, \ldots, q_{b_{\ell}}$ with $1 \leqslant b_{1}<b_{2}<\cdots<b_{\ell} \leqslant m$ such that

- for each $x \in\{1, \ldots, i\}$ and $y \in\{1,2, \ldots, \ell\}, s_{y}^{x}$ is adjacent to $q_{b_{y}}$ and non-adjacent to $q_{b_{z}}$ for all $z \in\{1, \ldots, \ell\} \backslash\{1, \ldots, y\}$.

We prove in Proposition 7.4 that the existence of this structure is guaranteed by assuming that the graph has no large wheel vertex-minor and the conditions that

- $C$ is sufficiently long,

- for every $v \in V(C), v$ has a neighbor in each $Y_{j}$,

- each vertex in $Y_{1} \cup Y_{2} \cup \cdots \cup Y_{i}$ has at most $n-1$ neighbors in $C$.

Concerning the final condition, if a vertex in $Y_{1} \cup Y_{2} \cup \cdots \cup Y_{i}$ has at least $n$ neighbors in $C$, then we can directly obtain a $W_{n}$ vertex-minor (Lemma 6.5).

Up until this point in the argument, we have made no assumptions on the possible edges between pairs of vertices in the set $S_{1} \cup S_{2} \cup \cdots \cup S_{i}$. As we argued in Figure 3, we want to find a large independent set formed by two disjoint subsets from two distinct sets $S_{j}$ and $S_{j^{\prime}}$. For this, we apply a Ramsey-type argument, which we call the rectangular Ramsey lemma (Proposition 5.2). This lemma implies that there exist a large subset $J \subseteq\{1,2, \ldots, \ell\}$ and $\left\{c_{1}, c_{2}\right\} \in\{1,2, \ldots, i\}$ such that $\left\{s_{x}^{c_{1}}: x \in J\right\} \cup\left\{s_{x}^{c_{2}}: x \in J\right\}$ is an independent set, if $G$ has no large clique.

We further refine the adjacency relations between $\left\{s_{x}^{c_{1}}: x \in J\right\} \cup\left\{s_{x}^{c_{2}}: x \in J\right\}$ and $C$ using the following Ramsey-type argument: for a graph $H$ on the vertex set $D \cup Y$ such that

- $H[D]$ is a sufficiently long induced cycle,

- for every $v \in D, v$ has a neighbor in $Y$, 

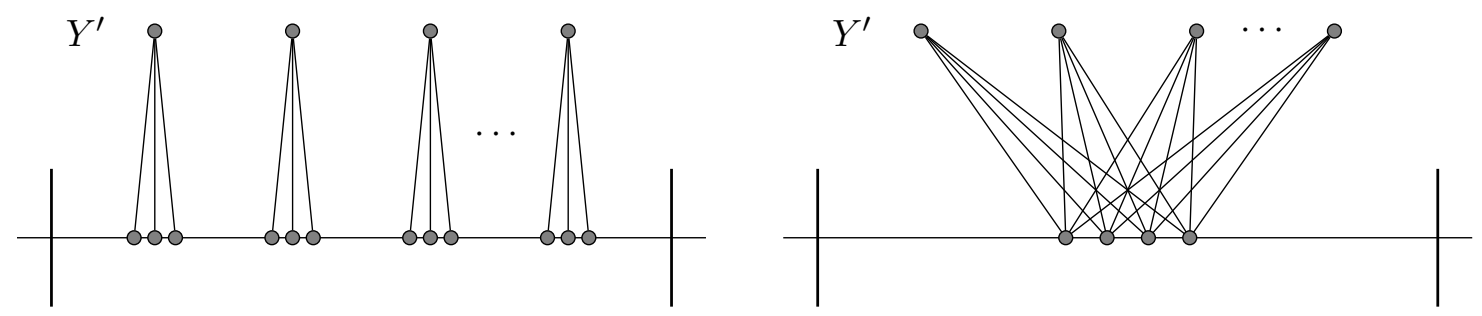

Figure 4: The intended application of the regular partition lemma.

- each vertex in $Y$ has at most $n-1$ neighbors in $D$,

there is a large subset $Y^{\prime} \subseteq Y$ and a partition of $H[D]$ into at most $n-1$ paths such that for each part, either vertices in $Y^{\prime}$ have the exactly same neighborhood, or neighborhoods appear in a consecutive order. Figure 4 shows the two cases for how the vertices of $Y^{\prime}$ can be adjacent to the vertices in $H[D]$ which is in one subpath of an element of the partition of $H[D]$. We prove this result in a more general setting, which we call the regular partition lemma (Proposition 4.2), with the hope that it might be of use in other situations.

Depending on the outcome of the application of the regular partition lemma, we show that $G$ contains a vertex-minor isomorphic to one of several cases we call a drum, a clam, and a hanging ladder, depicted in Figures 5, 6, 7, respectively.

\section{Regular partition lemma}

For a sequence $\left(A_{1}, \ldots, A_{\ell}\right)$ of finite subsets of an interval $I \subseteq \mathbb{R}$, a partition $\left\{I_{1}, \ldots, I_{k}\right\}$ of $I$ into intervals is called a regular partition of $I$ with respect to $\left(A_{1}, \ldots, A_{\ell}\right)$ if for all $i \in\{1, \ldots, k\}$, either

- $A_{1} \cap I_{i}=A_{2} \cap I_{i}=\cdots=A_{\ell} \cap I_{i} \neq \varnothing$, or

- $\left|A_{1} \cap I_{i}\right|=\left|A_{2} \cap I_{i}\right|=\cdots=\left|A_{\ell} \cap I_{i}\right|>0$, and for all $j, j^{\prime} \in\{1, \ldots, \ell\}$ with $j<j^{\prime}$, $\max \left(A_{j} \cap I_{i}\right)<\min \left(A_{j^{\prime}} \cap I_{i}\right)$, or

- $\left|A_{1} \cap I_{i}\right|=\left|A_{2} \cap I_{i}\right|=\cdots=\left|A_{\ell} \cap I_{i}\right|>0$, and for all $j, j^{\prime} \in\{1, \ldots, \ell\}$ with $j<j^{\prime}$, $\max \left(A_{j^{\prime}} \cap I_{i}\right)<\min \left(A_{j} \cap I_{i}\right)$.

The number of parts $k$ is called the order of the regular partition.

The following lemma is a strengthening of Erdős-Szekeres theorem. We simply follow the proof of Seidenberg [18]. We say that a sequence is identical if all elements of the sequence are same.

Lemma 4.1. For every sequence $\left(a_{1}, \ldots, a_{(\ell-1)^{3}+1}\right)$ of real numbers, there exists a subsequence $\left(a_{i_{1}}, \ldots, a_{i_{\ell}}\right)$ that is identical or strictly increasing or strictly decreasing.

Proof. For each $a_{i}$, we define a triplet $\left(a_{i}^{1}, a_{i}^{2}, a_{i}^{3}\right)$ where

- $a_{i}^{1}$ is the length of the longest identical subsequence ending at $a_{i}$,

- $a_{i}^{2}$ is the length of the longest strictly increasing subsequence ending at $a_{i}$, and

- $a_{i}^{3}$ is the length of the longest strictly decreasing subsequence ending at $a_{i}$. 
Note that $\left(a_{i}^{1}, a_{i}^{2}, a_{i}^{3}\right) \neq\left(a_{j}^{1}, a_{j}^{2}, a_{j}^{3}\right)$ for all $i \neq j$, since $a_{j}=a_{i}$ or $a_{j}>a_{i}$ or $a_{j}<a_{i}$. However, the number of different triplets such that $0<a_{i}^{1}, a_{i}^{2}, a_{i}^{3}<\ell$ is at most $(\ell-1)^{3}$. Therefore, there exists $a_{k}$ such that one of $a_{k}^{1}, a_{k}^{2}$, and $a_{k}^{3}$ is $\ell$, completing the proof.

Proposition 4.2 (Regular partition lemma). Let $I \subseteq \mathbb{R}$ be an interval. For all positive integers $k$ and $\ell$, there exists a positive integer $N=N(k, \ell)$ satisfying the following. For every sequence $\left(A_{1}, \ldots, A_{N}\right)$ of $k$-element subsets of $I$, there exist a subsequence $\left(A_{j_{1}}, \ldots, A_{j_{\ell}}\right)$ of $\left(A_{1}, \ldots, A_{N}\right)$ and a regular partition of $I$ with respect to $\left(A_{j_{1}}, \ldots, A_{j_{\ell}}\right)$ that has order at most $k$.

Proof. We recursively define $t(n, \ell), M(n, \ell), N(n, \ell)$ as follows

$$
\begin{aligned}
t(n, \ell) & = \begin{cases}\max \{N(i, N(n-i, \ell)): i=1,2, \ldots, n-1\} & \text { if } n>1, \\
\ell & \text { if } n=1 .\end{cases} \\
M(n, \ell) & =(t(n, \ell)-1)(\ell-1) n+1 . \\
N(n, \ell) & = \begin{cases}(M(n, \ell)-1)^{\left(2^{n}\right)}+1 & \text { if } n>1, \\
(\ell-1)^{3}+1 & \text { if } n=1 .\end{cases}
\end{aligned}
$$

We proceed by induction on $k$. If $k=1$, the statement is implied by Lemma 4.1, If $\ell=1$, then the partition $\{I\}$ of $I$ is a regular partition with respect to $A_{1}$. We may assume that $k, \ell \geqslant 2$. Note that $M(k, \ell) \geqslant t(k, \ell)$. By slightly abusing notation, let us identify $A_{i}$ with a strictly increasing sequence $\left(a_{i, 1}, a_{i, 2}, \ldots, a_{i, k}\right)$ of its elements. Let $M=M(k, \ell)$, and $t=t(k, \ell)$. Let $\mathcal{A}_{0}=\left(A_{1}, \ldots, A_{N}\right)$. For each $i=1,2, \ldots, k$, there exists a subsequence $\mathcal{A}_{i}$ of $\mathcal{A}_{i-1}$ such that the sequence of the $i$-th elements of terms of $\mathcal{A}_{i}$ is (not necessarily strictly) increasing or decreasing where

$$
\left|\mathcal{A}_{i}\right| \geqslant \sqrt{\left|\mathcal{A}_{i-1}\right|-1}+1
$$

by the Erdős-Szekeres Theorem. Then $\left|\mathcal{A}_{k}\right| \geqslant M$. Let $\mathcal{A}=\left(A_{i_{1}}, \ldots, A_{i_{M}}\right)$ be a subsequence of $\mathcal{A}_{k}$ of length $M$.

By the construction, for each $j \in\{1,2, \ldots, k\}$, the sequence $\left(a_{i_{1}, j}, a_{i_{2}, j}, \ldots, a_{i_{M}, j}\right)$ of the $j$-th elements of terms of $\mathcal{A}$ is increasing or decreasing. By symmetry, we may assume that $\left(a_{i_{1}, 1}, \ldots, a_{i_{M}, 1}\right)$ is increasing, because otherwise we consider the reverse $\left(A_{N}, A_{N-1}, \ldots, A_{1}\right)$.

Suppose that there exists an integer $0<j<k$ such that $\left(a_{i_{1}, j}, \ldots, a_{i_{M}, j}\right)$ is increasing and $\left(a_{i_{1}, j+1}, \ldots, a_{i_{M}, j+1}\right)$ is decreasing. Let $x \in\left(a_{i_{M}, j}, a_{i_{M}, j+1}\right)$. As $\left(a_{i_{1}, j}, \ldots, a_{i_{M}, j}\right)$ is increasing, the first $j$ elements of each term of $\mathcal{A}$ are less than $x$. Similarly the remaining $k-j$ elements of each term of $\mathcal{A}$ are greater than $x$ because $\left(a_{i_{1}, j+1}, \ldots, a_{i_{M}, j+1}\right)$ is decreasing. Thus we observe that

$$
\left|A_{i_{1}} \cap(-\infty, x]\right|=\left|A_{i_{2}} \cap(-\infty, x]\right|=\cdots=\left|A_{i_{M}} \cap(-\infty, x]\right|=j
$$

and

$$
\left|A_{i_{1}} \cap(x, \infty)\right|=\left|A_{i_{2}} \cap(x, \infty)\right|=\cdots=\left|A_{i_{M}} \cap(x, \infty)\right|=k-j .
$$

Since $0<j<k$ and $M \geqslant t \geqslant N(j, N(k-j, \ell))$, by the induction hypothesis applied to $\left(A_{i_{1}} \cap\right.$ $\left.(-\infty, x], A_{i_{2}} \cap(-\infty, x], \ldots, A_{i_{M}} \cap(-\infty, x]\right)$, there exist a subsequence $\mathcal{A}^{\prime}$ of $\mathcal{A}$ with $\left|\mathcal{A}^{\prime}\right|=N(k-j, \ell)$ and a regular partition of $I \cap(-\infty, x]$ with respect to $\mathcal{A}^{\prime}$ that has order at most $j$. Again by the induction hypothesis, we obtain a subsequence $\mathcal{A}^{\prime \prime}$ of $\mathcal{A}^{\prime}$ with $\left|\mathcal{A}^{\prime \prime}\right|=\ell$ and a regular partition of $I \cap(x, \infty)$ with respect to $\mathcal{A}^{\prime \prime}$ that has order at most $k-j$. The union of the regular partitions of 
$I \cap(-\infty, x]$ and $I \cap(x, \infty)$ is a regular partition of $I$ with respect to $\mathcal{A}^{\prime \prime}$ of order at most $k$, so we are done. Therefore, we may assume that $\left(a_{i_{1}, j}, \ldots, a_{i_{M}, j}\right)$ is increasing for every $j \in\{1, \ldots, k\}$.

Suppose that $a_{i_{s+t-1}, j}<a_{i_{s}, j+1}$ for some $1 \leqslant s \leqslant M-t+1$ and $1 \leqslant j \leqslant k-1$. Then there exists $x \in\left(a_{i_{s+t-1}, j}, a_{i_{s}, j+1}\right)$. We deduce that

$$
\left|A_{i_{s}} \cap(-\infty, x]\right|=\left|A_{i_{s+1}} \cap(-\infty, x]\right|=\cdots=\left|A_{i_{s+t-1}} \cap(-\infty, x]\right|=j
$$

and

$$
\left|A_{i_{s}} \cap(x, \infty)\right|=\left|A_{i_{s+1}} \cap(x, \infty)\right|=\cdots=\left|A_{i_{s+t-1}} \cap(x, \infty)\right|=k-j .
$$

Since $t \geqslant N(j, N(k-j, \ell))$, by applying the induction hypothesis to a partition $I \cap(-\infty, x]$ and to a partition $I \cap(x, \infty)$ as in the previous paragraph, we are done.

Thus, we may assume that $a_{i_{s+t-1}, j} \geqslant a_{i_{s}, j+1}$ for all $1 \leqslant s \leqslant M-t+1$ and $1 \leqslant j \leqslant k-1$. Therefore $a_{i_{s+(t-1) k}, 1} \geqslant a_{i_{s+t-1}, k}>a_{i_{s+t-1}, k-1} \geqslant a_{i_{s}, k}$ for all $s$ with $1 \leqslant s \leqslant M-(t-1) k$. This implies that $\max A_{i_{1+(t-1) k j}}<\min A_{i_{1+(t-1) k(j+1)}}$ for each $0 \leqslant j \leqslant \ell-2$ and therefore $\{I\}$ is a regular partition of $I$ with respect to $\left(A_{i_{1}}, A_{i_{1+(t-1) k}}, \ldots, A_{i_{1+(t-1) k(\ell-1)}}\right)$.

Corollary 4.3. Let $I$ be an interval in $\mathbb{R}$. For all positive integers $k$ and $\ell$, there exists an integer $N=N^{\prime}(k, \ell)$ satisfying the following: For every sequence $\left(A_{1}, \ldots, A_{N}\right)$ of sets of at most $k$ reals in $I$, there exist a sequence $1 \leqslant j_{1}<j_{2}<\cdots<j_{\ell} \leqslant N$ and a regular partition of $I$ with respect to $\left(A_{j_{1}}, \ldots, A_{j_{\ell}}\right)$ that has order at most $k$.

\section{Rectangular Ramsey Lemma}

Let $G$ be a graph with vertex set $\{1,2, \ldots, m\} \times\{1,2, \ldots, n\}$. We would like to show that either $G$ has a large clique or there exist subsets $X \subseteq\{1,2, \ldots, m\}$ and $Y \subseteq\{1,2, \ldots, n\}$ such that $X \times Y$ is an independent set in $G$ and both $|X|$ and $|Y|$ are large. We prove it using the Product Ramsey Theorem. For a set $X$ and a non-negative integer $k$, we denote by $\left(\begin{array}{l}X \\ k\end{array}\right)$ the set of all $k$-element subsets of $X$.

Theorem 5.1 (Theorem 11.5 of [19]; See also [12]). Let $r, t$ be positive integers, and let $k_{1}, k_{2}, \ldots, k_{t}$ be nonnegative integers, and let $m_{1}, m_{2}, \ldots, m_{t}$ be integers with $m_{i} \geqslant k_{i}$ for each $i \in\{1,2, \ldots, t\}$. Then there exists an integer $R=R_{\text {prod }}\left(r, t ; k_{1}, k_{2}, \ldots, k_{t} ; m_{1}, m_{2}, \ldots, m_{t}\right)$ such that if $X_{1}, X_{2}, \ldots, X_{t}$ are sets with $\left|X_{i}\right| \geqslant R$ for each $i \in\{1,2, \ldots, t\}$, then for every function $f:\left(\begin{array}{l}X_{1} \\ k_{1}\end{array}\right) \times\left(\begin{array}{l}X_{2} \\ k_{2}\end{array}\right) \times$ $\cdots \times\left(\begin{array}{c}X_{t} \\ k_{t}\end{array}\right) \rightarrow\{1,2, \ldots, r\}$, there exist an element $\alpha \in\{1,2, \ldots r\}$ and subsets $Y_{1}, Y_{2}, \ldots, Y_{t}$ of $X_{1}, X_{2}, \ldots, X_{t}$, respectively, so that $\left|Y_{i}\right| \geqslant m_{i}$ for each $i \in\{1,2, \ldots, t\}$, and $f$ maps every element of $\left(\begin{array}{l}Y_{1} \\ k_{1}\end{array}\right) \times\left(\begin{array}{l}Y_{2} \\ k_{2}\end{array}\right) \times \cdots \times\left(\begin{array}{l}Y_{t} \\ k_{t}\end{array}\right)$ to $\alpha$.

Proposition 5.2. For all positive integers $a, b$, and $k$, there exist positive integers $M=R_{1}(a, b, k)$ and $N=R_{2}(a, b, k)$ such that for all $m \geqslant M$ and $n \geqslant N$, every graph $G$ on $\{1,2, \ldots, m\} \times$ $\{1,2, \ldots, n\}$ either has a clique of $k$ vertices or has subsets $X \subseteq\{1,2, \ldots, m\}$ and $Y \subseteq\{1,2, \ldots, n\}$ such that $|X|=a,|Y|=b$, and $X \times Y$ is an independent set in $G$.

Proof. Let $t:=\max (a, b, k)$, and let $M=N=R_{\text {prod }}(7,2 ; 2,2 ; t, t)$. We may assume that $G$ is a graph on $\{1,2, \ldots, M\} \times\{1,2, \ldots, N\}$. Let us write $v_{i j}=(i, j)$ to denote a vertex.

We define a function $f:\left(\begin{array}{c}\{1,2, \ldots, M\} \\ 2\end{array}\right) \times\left(\begin{array}{c}\{1,2, \ldots, N\} \\ 2\end{array}\right) \rightarrow\{1,2, \ldots, 7\}$ as follows. For $\left\{x_{1}, x_{2}\right\} \subseteq$ $\{1,2, \ldots, M\}$ and $\left\{y_{1}, y_{2}\right\} \subseteq\{1,2, \ldots, N\}$ with $x_{1}<x_{2}$ and $y_{1}<y_{2}$, let

$$
\left(e_{1}, e_{2}, e_{3}, e_{4}, e_{5}, e_{6}\right):=\left(v_{x_{1} y_{1}} v_{x_{2} y_{1}}, v_{x_{1} y_{1}} v_{x_{1} y_{2}}, v_{x_{1} y_{1}} v_{x_{2} y_{2}}, v_{x_{2} y_{1}} v_{x_{1} y_{2}}, v_{x_{2} y_{1}} v_{x_{2} y_{2}}, v_{x_{1} y_{2}} v_{x_{2} y_{2}}\right),
$$


and let $f\left(\left\{x_{1}, x_{2}\right\},\left\{y_{1}, y_{2}\right\}\right):=i$ if $G$ contains $e_{i}$ but does not contain $e_{j}$ for all $j<i$, and $f\left(\left\{x_{1}, x_{2}\right\},\left\{y_{1}, y_{2}\right\}\right):=7$ if $G$ contains no edges in $\left\{e_{1}, \ldots, e_{6}\right\}$. By Theorem 5.1, there exist $\alpha \in\{1,2, \ldots, 7\}, X \subseteq\{1,2, \ldots, M\}$, and $Y \subseteq\{1,2, \ldots, N\}$ with $|X| \geqslant t$ and $|Y| \geqslant t$ such that $f$ maps every element of $\left(\begin{array}{c}X \\ 2\end{array}\right) \times\left(\begin{array}{c}Y \\ 2\end{array}\right)$ to $\alpha$. If $\alpha=7$, then $X \times Y$ is an independent set with $|X| \geqslant a$ and $|Y| \geqslant b$, and if $\alpha \in\{1,2, \ldots, 6\}$, then $G$ contains a clique of size $t \geqslant k$.

\section{$6 \quad$ Manufacturing wheels}

We will use the following Ramsey-type result on connected graphs.

Theorem 6.1 (folklore; see Diestel [9]). For $k \geqslant 1$ and $\ell \geqslant 3$, every connected graph on at least $k^{\ell-2}+1$ vertices contains a vertex of degree at least $k$ or an induced path on $\ell$ vertices.

The following lemma is useful to find an induced matching in a bipartite graph.

Lemma 6.2 (Lemma 7.8 of [16]). Let $n$ be a positive integer. Let $G$ be a bipartite graph with a bipartition $(A, B)$ such that

- every vertex in A has a neighbor,

- every vertex in $B$ has at most $n$ neighbors.

Then there is an induced matching of size at least $|A| / n$.

For every integer $n \geqslant 3$, let $\mu(n)=(n-1)\left(R(n, n)^{2 n-3}+1\right)$. Lemma 6.4 is useful to reduce the size of a connected subgraph.

Lemma 6.3 (Choi, Kwon, and Oum [7]). Let $H$ be a connected graph with at least 2 vertices. For each vertex $v$ of $H$, either $H-v$ or $H * v-v$ is connected.

Lemma 6.4. Let $n \geqslant 3$ be an integer and let $G$ be a graph on the vertex set $A \cup U \cup S$ such that

1. A, $U$, and $S$ are pairwise disjoint,

2. there are no edges between $A$ and $S$,

3. $U$ is an independent set,

4. each vertex in $U$ has a neighbor on $S$, and

5. there exists a vertex $w \in S$ where for every $v \in N_{G}(U) \cap S$, there is a path $P$ from $v$ to $w$ in $G[S]$ with $N_{G}(U) \cap V(P)=\{v\}$.

If $|U| \geqslant \mu(n)$, then there exist $U^{\prime} \subseteq U$ with $\left|U^{\prime}\right| \geqslant n$ and $v \in S$ and a graph $G^{\prime}$ on $A \cup U^{\prime} \cup\{v\}$ such that

1. $G^{\prime}\left[A \cup U^{\prime}\right]=G\left[A \cup U^{\prime}\right]$,

2. $v$ is adjacent to all vertices in $U^{\prime}$ and has no neighbors in $A$ in $G^{\prime}$,

3. $G^{\prime}$ is a vertex-minor of $G$. 
Proof. Let $m:=\mu(n)$ and let $U:=\left\{u_{1}, u_{2}, \ldots, u_{m}\right\}$. For each $v \in N_{G}(U) \cap S$, let $P_{v}$ be a path from $v$ to $w$ in $G[S]$ with $N_{G}(U) \cap V\left(P_{v}\right)=\{v\}$.

Suppose $w \in N_{G}(U) \cap S$. By the assumption that for every $v \in N_{G}(U) \cap S$ it holds that $N_{G}(U) \cap V\left(P_{v}\right)=\{v\}$, we conclude that $N_{G}(U) \cap S=\{w\}$. Therefore, $w$ is adjacent to all vertices in $U$, and we are done. We may assume that $w \in S \backslash N_{G}(U)$.

If there is a vertex in $S$ having at least $n$ neighbors on $U$, then we are done. We may assume that every vertex in $S$ has at most $n-1$ neighbors on $U$. By Lemma 6.2, there exist a subset $\left\{a_{1}, a_{2}, \ldots, a_{m /(n-1)}\right\}$ of $\{1,2, \ldots, m\}$ and a subset $\left\{s_{1}, s_{2}, \ldots, s_{m /(n-1)}\right\}$ of $S$ such that

- $u_{a_{i}}$ is adjacent to $s_{j}$ if and only if $i=j$.

Let $U_{1}:=A \cup\left\{u_{a_{i}}: 1 \leqslant i \leqslant m /(n-1)\right\}$ and $U_{2}:=\left\{s_{i}: 1 \leqslant i \leqslant m /(n-1)\right\}$. Let $G_{1}:=$ $G\left[U_{1} \cup U_{2} \cup\left(\bigcup_{v \in U_{2}} V\left(P_{v}\right)\right)\right]$. Note that $N_{G_{1}}\left(U_{1}\right)=U_{2}, G_{1}-U_{1}$ is connected, and every vertex in $V\left(G_{1}\right) \backslash\left(U_{1} \cup U_{2}\right)$ has no neighbors in $U_{1}$.

Choose a sequence of graphs $H_{1}, H_{2}, \ldots, H_{y}$ such that

(1) $H_{1}=G_{1}$,

(2) $V\left(H_{y}\right)=U_{1} \cup U_{2}$,

(3) for each $i \in\{1,2, \ldots, y-1\}, H_{i+1}=H_{i}-v_{i}$ or $H_{i+1}=H_{i} * v_{i}-v_{i}$ for some $v_{i} \in V\left(H_{i}\right) \backslash\left(U_{1} \cup U_{2}\right)$,

(4) for each $i \in\{1,2, \ldots, y\}, H_{i}-U_{1}$ is connected.

We claim that such a sequence always exists. Let $H_{1}, H_{2}, \ldots, H_{y^{\prime}}$ be a maximal sequence satisfying (1), (3), and (4). Assume, to reach a contradiction, that $V\left(H_{y^{\prime}}\right) \neq U_{1} \cup U_{2}$. By the assumptions, we have $U_{1} \cup U_{2} \subseteq V\left(H_{y^{\prime}}\right)$, and therefore, $V\left(H_{y^{\prime}}\right) \backslash\left(U_{1} \cup U_{2}\right) \neq \varnothing$. Let $v_{y^{\prime}} \in V\left(H_{y^{\prime}}\right) \backslash\left(U_{1} \cup U_{2}\right)$. Since $H_{y^{\prime}}-U_{1}$ is connected, by Lemma 6.3, $\left(H_{y^{\prime}}-U_{1}\right)-v_{y^{\prime}}$ or $\left(H_{y^{\prime}}-U_{1}\right) * v_{y^{\prime}}-v_{y^{\prime}}$ is connected. We fix $H_{y^{\prime}+1}$ to be one of $\left(H_{y^{\prime}}-U_{1}\right)-v_{y^{\prime}}$ and $\left(H_{y^{\prime}}-U_{1}\right) * v_{y^{\prime}}-v_{y^{\prime}}$ which is connected. This contradicts the maximality of the sequence. We conclude that there exists a sequence $H_{1}, H_{2}, \ldots, H_{y}$ satisfying (1) - (4). Let $G_{2}:=H_{y}$. Note that

- $V\left(G_{2}\right)=U_{1} \cup U_{2}$,

- $G_{2}\left[U_{2}\right]$ is connected,

- $G_{2}-E\left(G_{2}\left[U_{2}\right]\right)=G_{1}\left[U_{1} \cup U_{2}\right]-E\left(G_{1}\left[U_{2}\right]\right)=G\left[U_{1} \cup U_{2}\right]-E\left(G\left[U_{2}\right]\right)$.

Since $\left|U_{2}\right|=m /(n-1)=R(n, n)^{2 n-3}+1$, by Theorem 6.1, $G_{2}\left[U_{2}\right]$ contains either a vertex of degree at least $R(n, n)$ or an induced path on $2 n-1$ vertices.

Suppose $G_{2}\left[U_{2}\right]$ contains a vertex $s_{j}$ of degree at least $R(n, n)$ for some $1 \leqslant j \leqslant m /(n-1)$. Since $s_{j}$ has at least $R(n, n)$ neighbors in $U_{2}, N_{G_{2}}\left(s_{j}\right) \cap U_{2}$ contains either a clique of size $n$ or an independent set of size $n$. We define

$$
G_{3}:= \begin{cases}G_{2}-u_{a_{j}} & \text { if } N_{G_{2}}\left(s_{j}\right) \cap U_{2} \text { contains an independent set of size } n \\ \left(G_{2}-u_{a_{j}}\right) * s_{j} & \text { otherwise. }\end{cases}
$$

Note that $N_{G_{3}}\left(s_{j}\right) \cap U_{2}$ contains an independent set of size $n$. Let $\left\{s_{d_{1}}, s_{d_{2}}, \ldots, s_{d_{n}}\right\}$ be an independent set in $N_{G_{3}}\left(s_{j}\right) \cap U_{2}$. Note that the application of a local complementation when we obtain 
$G_{3}$ does not change the adjacency relation between $\left\{s_{d_{1}}, s_{d_{2}}, \ldots, s_{d_{n}}\right\}$ and $\left\{u_{a_{d_{1}}}, u_{a_{d_{2}}}, \ldots, u_{a_{d_{n}}}\right\}$ as $s_{j}$ has no neighbors on $\left\{u_{a_{d_{1}}}, u_{a_{d_{2}}}, \ldots, u_{a_{d_{n}}}\right\}$ in $G_{2}$. Let

$$
G^{\prime}:=\left(G_{3} * s_{d_{1}} * s_{d_{2}} * \cdots * s_{d_{n}}\right)\left[A \cup\left\{u_{a_{d_{i}}}: 1 \leqslant i \leqslant n\right\} \cup\left\{s_{j}\right\}\right] .
$$

Then we have $G^{\prime}\left[A \cup\left\{u_{a_{d_{i}}}: 1 \leqslant i \leqslant n\right\}\right]=G\left[A \cup\left\{u_{a_{d_{i}}}: 1 \leqslant i \leqslant n\right\}\right]$, and $s_{j}$ is adjacent to all vertices in $\left\{u_{a_{d_{i}}}: 1 \leqslant i \leqslant n\right\}$ and has no neighbors in $A$ in $G^{\prime}$, as required.

Suppose $G_{2}\left[U_{2}\right]$ contains an induced path $s_{i_{1}} s_{i_{2}} \ldots s_{i_{2 n-1}}$. Let

$$
G^{\prime}:=\left(G_{3} * s_{i_{1}} * s_{i_{2}} * \cdots * s_{i_{2 n-2}}\right)-\left\{s_{i_{1}}, s_{i_{2}}, \ldots, s_{i_{2 n-2}}\right\}-\left\{u_{i_{2}}, u_{i_{4}}, u_{i_{6}}, \ldots, u_{i_{2 n-2}}\right\} .
$$

Then in $G^{\prime}, s_{i_{2 n-1}}$ is adjacent to all of $u_{i_{1}}, u_{i_{3}}, u_{i_{5}}, \ldots, u_{i_{2 n-1}}$, and $\left\{u_{i_{1}}, u_{i_{3}}, u_{i_{5}}, \ldots, u_{i_{2 n-1}}\right\}$ is an independent set of size $n$, and $s_{i_{2 n-1}}$ has no neighbor in $A$, as required.

\subsection{From a partial wheel with many spokes}

Lemma 6.5. Let $n \geqslant 3$ be an integer and let $G$ be a graph such that $G-v$ is an induced cycle of length at least $n+3$. If the degree of $v$ is at least $n$, then $G$ has $W_{n}$ as a vertex-minor.

Proof. Let $s$ be the length of the induced cycle $G-v$. Let $v_{1}, \ldots, v_{s}$ be the vertices of the cycle in a cyclic order and let $v_{s+\ell}:=v_{\ell}$ for $1 \leqslant \ell \leqslant s$. Let $t$ be the degree of $v$. Note that $s \geqslant t$.

We prove by induction on $s+t$. The following statements cover base cases which are either $t=n$ or $s=n+3$ :

- (Case 1. $t=n$.) Let $v_{i_{1}}, v_{i_{2}}, \ldots, v_{i_{s-t}}$ be the vertices of $G-v$ that are non-adjacent to $v$ in $G$. The graph obtained from $G$ by smoothing $v_{i_{1}}, v_{i_{2}}, \ldots, v_{i_{s_{t}}}$ is isomorphic to $W_{n}$.

- (Case 2. $s=n+3$ and $t=n+1$.) Let $v_{i}, v_{j}$ be the vertices in $G-v$ that are non-adjacent to $v$. Note that we may assume that $i \neq j+1$ and $i \neq j+2$ by symmetry. The graph $\left(G * v_{i} * v_{j+1} * v_{j+2}\right)-\left\{v_{i}, v_{j+1}, v_{j+2}\right\}$ is isomorphic to $W_{n}$.

- (Case 3. $s=n+3$ and $t=n+2$.) Let $v_{i}$ be the vertex in $G-v$ that is non-adjacent to $v$. The graph $\left(G * v_{i+1} * v_{i} * v_{i-1}\right)-\left\{v_{i-1}, v_{i}, v_{i+1}\right\}$ is isomorphic to $W_{n}$.

- (Case 4. $s=n+3$ and $t=n+3$.) Let $v_{i}$ be a vertex in $G-v$. The graph $\left(G * v_{i} * v_{i-1} *\right.$ $\left.v_{i+1}\right)-\left\{v_{i-1}, v_{i}, v_{i+1}\right\}$ is isomorphic to $W_{n}$.

We may assume that $s>n+3$ and $t>n$. Suppose that $s>t$. There exists $i$ such that $v$ is not adjacent to $v_{i}$ and adjacent to $v_{i+1}$. Let $G_{1}=G * v_{i}-v_{i}$. Then $G_{1}-v$ is an induced cycle of length $s-1 \geqslant n+3$ and the degree of $v$ is $t$ in $G_{1}$. By induction hypothesis, $G_{1}$ has $W_{n}$ as a vertex-minor, which implies that $G$ has $W_{n}$ as a vertex-minor. Now, we may assume that $s=t>n+3$. For a vertex $u$ in $G-v$, let $G_{2}=G * u-u$. Then $G_{2}-v$ is an induced cycle of length $s-1 \geqslant n+3$ and the degree of $v$ is $t-3 \geqslant n$ in $G_{2}$. By induction hypothesis, $G_{2}$ has $W_{n}$ as a vertex-minor, which implies that $G$ has $W_{n}$ as a vertex-minor.

\subsection{From drums, clams, and hanging ladders}

A drum on $3 n$ vertices is the graph on the vertex set $\left\{v_{1}, v_{2}, \ldots, v_{n}, w_{1}, w_{2}, \ldots, w_{n}, u_{1}, u_{2}, \ldots, u_{n}\right\}$ such that $v_{1} v_{2} \cdots v_{n}$ is an induced path, $w_{1} w_{2} \cdots w_{n}$ is an induced cycle, each $u_{i}$ is adjacent only to $v_{i}$ and $w_{i}$, and there are no edges between $\left\{v_{1}, v_{2}, \ldots, v_{n}\right\}$ and $\left\{w_{1}, w_{2}, \ldots, w_{n}\right\}$. See Figure 5 for an illustration. 


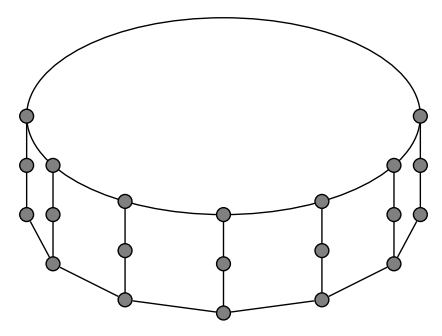

Figure 5: A drum

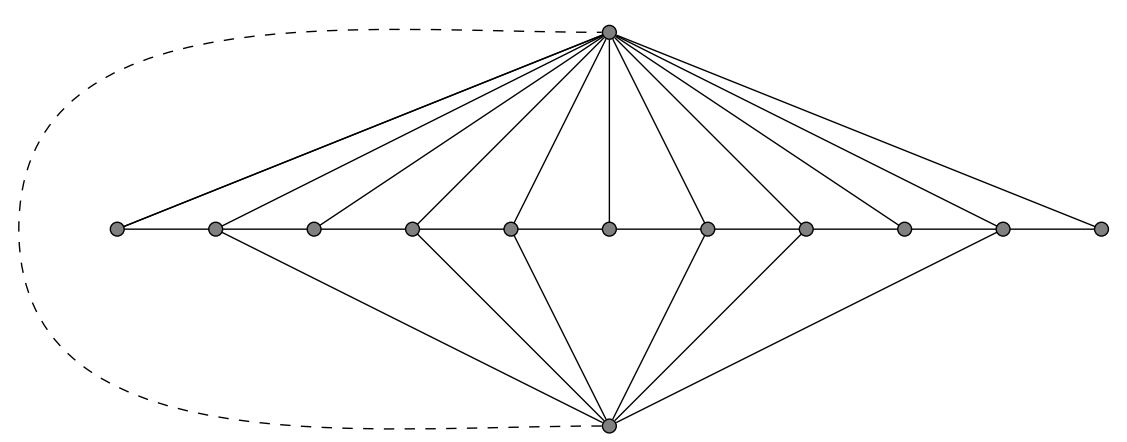

Figure 6: A clam. (A dashed line may be an edge or not.)

Lemma 6.6. For an integer $n \geqslant 3$, a drum on $3(2 n-1)$ vertices has $W_{n}$ as a vertex-minor.

Proof. Let $G$ be a drum on 3(2n-1) vertices with the vertex labels as in the definition of drums. Let $H=G * v_{1} * v_{2} * v_{3} \cdots * v_{2 n-2}$. Then, in $H, v_{2 n-1}$ is adjacent to all of $u_{1}, u_{2}, \ldots, u_{2 n-1}$. Furthermore $\left\{u_{1}, u_{3}, u_{5}, \ldots, u_{2 n-1}\right\}$ is an independent set in $H$. Thus,

$$
H-\left\{u_{2}, u_{4}, u_{6}, \ldots, u_{2 n-2}, v_{1}, v_{2}, \ldots, v_{2 n-2}\right\}
$$

is isomorphic to a subdivision of $W_{n}$.

For an integer $n \equiv 1(\bmod 3)$, a clam on $n$ vertices is a graph on the vertex set $\left\{v_{1}, v_{2}, \ldots, v_{n-2}, h_{1}, h_{2}\right\}$ such that $v_{1} v_{2} \ldots v_{n-2}$ is an induced path, $h_{1}$ is adjacent to all of $v_{1}, v_{2}, \ldots, v_{n-2}$, and $h_{2}$ is adjacent to $v_{i}$ if and only if $1<i<n-2$ and $i \not \equiv 0(\bmod 3)$. Note that $h_{1}$ and $h_{2}$ may be adjacent in a clam.

Lemma 6.7. For an integer $n \geqslant 2$, a clam on $3 n+4$ vertices contains $W_{2 n}$ or $W_{2 n+1}$ as a vertex-minor.

Proof. Let $G$ be a clam on $3 n+4$ vertices with the vertex labels as in the definition. Let $H=G * v_{3} * v_{6} * v_{9} * \cdots * v_{3 n}$. In $H, h_{1}$ is non-adjacent to $\left\{v_{2}, v_{4}, v_{5}, v_{7}, \ldots, v_{3 n-1}, v_{3 n+1}\right\}$, and $h_{1} v_{1} v_{2} v_{4} v_{5} v_{7} v_{8} \cdots v_{3 n-1} v_{3 n+1} v_{3 n+2} h_{1}$ is an induced cycle of length $2 n+3$. Still in $H, h_{2}$ is adjacent to $2 n$ vertices among $v_{1}, v_{2}, v_{4}, v_{5}, v_{7}, v_{8}, \ldots, v_{3 n-1}, v_{3 n+1}, v_{3 n+2}$. Thus, $H-\left\{v_{3}, v_{6}, v_{9}, \cdots, v_{3 n}\right\}$ is isomorphic to a subdivision of $W_{2 n}$ or $W_{2 n+1}$, depending on whether or not $h_{2}$ is adjacent to $h_{1}$ in $H$. 


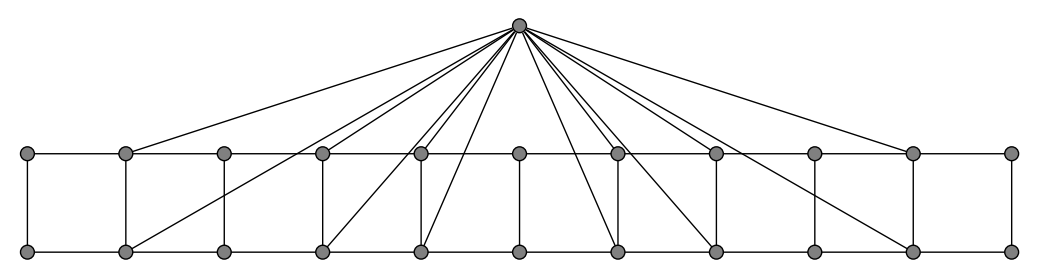

Figure 7: A hanging ladder

A hanging ladder on $6 n+5$ vertices is a graph on the vertex set $\left\{v_{1}, v_{2}, \ldots, v_{3 n+2}, w_{1}, w_{2}, \ldots, w_{3 n+2}, c\right\}$ such that

- $v_{i}$ is adjacent to $w_{j}$ if and only if $i=j$,

- $v_{1} v_{2} \ldots v_{3 n+2}$ and $w_{1} w_{2} \ldots w_{3 n+2}$ are induced paths,

- the set of neighbors of $c$ is $\left\{v_{i}, w_{i}: 1<i<3 n+2, i \not \equiv 0(\bmod 3)\right\}$.

Lemma 6.8. For an integer $n \geqslant 2$, a hanging ladder on $6 n+5$ vertices contains $W_{4 n}$ as a vertexminor.

Proof. Let $G$ be the hanging ladder on $6 n+5$ vertices with the labels as in the definition. Let $H=G \wedge v_{3} w_{3} \wedge v_{6} w_{6} \wedge \cdots \wedge v_{3 n} w_{3 n}$. Then the vertex set $\left\{v_{i}, w_{i}: 1 \leqslant i \leqslant 3 n+2, i \neq \equiv 0(\bmod 3)\right\}$ induces a cycle in $H$. As $c$ is still adjacent to $4 n$ vertices on this cycle, $H$ is isomorphic to a subdivision of $W_{4 n}$.

\subsection{From extended drums}

An extended drum of order $n$ is a graph $G$ on the vertex set $\left\{w_{1}, w_{2}, \ldots, w_{n}, u_{1}, u_{2}, \ldots, u_{n}\right\} \cup S$ such that

- $S$ and $\left\{w_{1}, w_{2}, \ldots, w_{n}, u_{1}, u_{2}, \ldots, u_{n}\right\}$ are disjoint,

- $w_{1} w_{2} \cdots w_{n} w_{1}$ is an induced cycle,

- $\left\{u_{1}, u_{2}, \ldots, u_{n}\right\}$ is an independent set,

- $u_{i}$ is adjacent to $w_{j}$ if and only if $i=j$,

- each $u_{i}$ has a neighbor in $S$,

- there are no edges between $S$ and $\left\{w_{1}, \ldots, w_{n}\right\}$.

- there exists a vertex $w \in S$ where for every $v \in N_{G}\left(\left\{u_{1}, u_{2}, \ldots, u_{n}\right\}\right) \cap S$, there is a path $P$ from $v$ to $w$ in $G[S]$ with $N_{G}\left(\left\{u_{1}, u_{2}, \ldots, u_{n}\right\}\right) \cap V(P)=\{v\}$.

Lemma 6.9. For an integer $n \geqslant 3$, an extended drum of order $\mu(n)$ has $W_{n}$ as a vertex-minor.

Proof. Let $G$ be an extended drum of order $\mu(n)$. By Lemma 6.4, there exist $U \subseteq\left\{u_{1}, u_{2}, \ldots, u_{\mu(n)}\right\}$ with $|U| \geqslant n$ and $v \in S$ and a graph $G^{\prime}$ on $\left\{w_{1}, w_{2}, \ldots, w_{\mu(n)}\right\} \cup U \cup\{v\}$ such that

- $G^{\prime}\left[\left\{w_{1}, w_{2}, \ldots, w_{\mu(n)}\right\} \cup U\right]=G\left[\left\{w_{1}, w_{2}, \ldots, w_{\mu(n)}\right\} \cup U\right]$ 
- $v$ is adjacent to all vertices in $U$ and has no neighbors in $\left\{w_{1}, w_{2}, \ldots, w_{\mu(n)}\right\}$ in $G^{\prime}$,

- $G^{\prime}$ is a vertex-minor of $G$.

Then $G^{\prime}$ is a subdivision of $W_{n}$, and therefore, $G$ contains a vertex-minor isomorphic to $W_{n}$.

\subsection{From extended clams}

An extended clam of order $n$ is a graph $G$ on the vertex set

$$
\left\{p_{1}, p_{2}, \ldots, p_{2 n}, v_{1}, v_{2}, \ldots, v_{n}, w_{1}, w_{2}, \ldots, w_{n}, h\right\} \cup S
$$

such that

- $S$ and $\left\{p_{1}, p_{2}, \ldots, p_{2 n}, v_{1}, v_{2}, \ldots, v_{n}, w_{1}, w_{2}, \ldots, w_{n}, h\right\}$ are disjoint,

- $p_{1} p_{2} \cdots p_{2 n}$ is an induced path,

- $\left\{v_{1}, \ldots, v_{n}, w_{1}, \ldots, w_{n}\right\}$ is an independent set,

- $v_{i}$ is adjacent to $p_{j}$ if and only if $j=2 i-1$,

- $w_{i}$ is adjacent to $p_{j}$ if and only if $j=2 i$,

- $h$ is adjacent to all vertices in $\left\{v_{1}, \ldots, v_{n}\right\}$, but non-adjacent to $\left\{p_{1}, \ldots, p_{2 n}\right\} \cup S$,

- each $w_{i}$ has a neighbor in $S$, and there are no edges between $S$ and $\left\{v_{1}, \ldots, v_{n}, p_{1}, \ldots, p_{2 n}\right\}$,

- there exists a vertex $w \in S$ where for every $v \in N_{G}\left(\left\{w_{1}, w_{2}, \ldots, w_{n}\right\}\right) \cap S$, there is a path $P$ from $v$ to $w$ in $G[S]$ with $N_{G}\left(\left\{w_{1}, w_{2}, \ldots, w_{n}\right\}\right) \cap V(P)=\{v\}$.

The simple extended clam is an extended clam such that $S$ consists of one vertex $z$ that is adjacent to all vertices in $\left\{w_{1}, \ldots, w_{n}\right\}$ and $h$ is adjacent to all vertices in $\left\{w_{1}, \ldots, w_{n}\right\}$.

Lemma 6.10. For an integer $n \geqslant 2$, the simple extended clam of order $2 n+1$ contains a clam on $3 n+4$ vertices as a vertex-minor, and thus contains $W_{2 n}$ or $W_{2 n+1}$ as a vertex-minor.

Proof. Let $G$ be the simple extended clam of order $2 n+1$, and let $G_{1}:=G * w_{1} * w_{2} * w_{3} * \cdots * w_{2 n}$. In $G_{1}$, both $z$ and $h$ are adjacent to $p_{2 i}$ for all $i \in\{1, \ldots, 2 n\}$. Then

$$
G_{1}-\left\{w_{1}, w_{2}, \ldots, w_{2 n+1}, v_{3}, v_{5}, \ldots, v_{2 n-1}, p_{4 n+2}\right\}
$$

is a subdivision of a clam on $(4 n+2)-(n-1+1)+2=3 n+4$ vertices. By Lemma 6.7, it contains a vertex-minor isomorphic to $W_{2 n}$ or $W_{2 n+1}$.

Lemma 6.11. For an integer $n \geqslant 3$, an extended clam of order $\mu(n)+\mu(2 n+1)-1$ contains $W_{n}$ as a vertex-minor.

Proof. Let $G$ be an extended clam or order $\mu(n)+\mu(2 n+1)-1$. There exists $I \subseteq\{1, \ldots, \mu(n)+$ $\mu(2 n+1)-1\}$ such that either

- $|I| \geqslant \mu(n)$ and $h$ is anti-complete to $\left\{w_{i}: i \in I\right\}$, or 


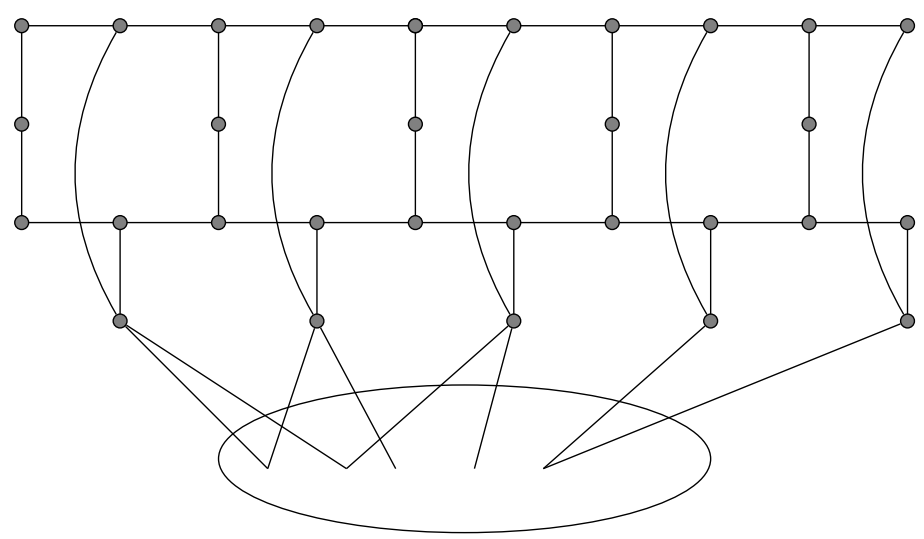

Figure 8: A simple extended hanging ladder of order 5.

- $|I| \geqslant \mu(2 n+1)$ and $h$ is complete to $\left\{w_{i}: i \in I\right\}$.

When $|I| \geqslant \mu(n)$ and $h$ is anti-complete to $\left\{w_{i}: i \in I\right\}, G$ contains a subdivision of an extended drum of order $\mu(n)$, with the cycle $h v_{1} p_{1} p_{2} \ldots p_{(2 \mu(n)+2 \mu(2 n+1)-3)} v_{(\mu(n)+\mu(2 n+1)-1)} h$. Then by Lemma 6.9, it contains a vertex-minor isomorphic to $W_{n}$. We may assume that $|I| \geqslant \mu(2 n+1)$ and $h$ is complete to $\left\{w_{i}: i \in I\right\}$. Let $G_{1}:=G-\left\{v_{i}, w_{i}: i \in\{1,2, \ldots, \mu(n)+\mu(2 n+1)-1\} \backslash I\right\}$, and let $A:=V\left(G_{1}\right) \backslash\left(\left\{w_{i}: i \in I\right\} \cup S\right)$.

By Lemma 6.4, there exist $U \subseteq\left\{w_{i}: i \in I\right\}$ with $|U| \geqslant 2 n+1$ and $v \in S$ and a graph $G_{2}$ on $A \cup U \cup\{v\}$ such that

- $G_{2}[A \cup U]=G[A \cup U]$,

- $v$ is adjacent to all vertices in $U$ and has no neighbors in $A$ in $G_{2}$,

- $G_{2}$ is a vertex-minor of $G$.

Then $G_{2}$ is a subdivision of the simple extended clam of order $2 n+1$, and therefore, $G$ contains a vertex-minor isomorphic to $W_{2 n}$ or $W_{2 n+1}$ by Lemma 6.10.

\subsection{From extended hanging ladders}

For integers $t, n \geqslant 2$, a $t$-extended hanging ladder of order $n$ is a graph $G$ on the vertex set

$$
\left\{p_{1}, p_{2}, \ldots, p_{2 n}, v_{1}, v_{2}, \ldots, v_{n}, w_{1}, w_{2}, \ldots, w_{n}\right\} \cup\left\{q_{1}, q_{2}, \ldots, q_{r}\right\} \cup S
$$

for some $r$ such that

- $S$ and $\left\{p_{1}, p_{2}, \ldots, p_{2 n}, v_{1}, v_{2}, \ldots, v_{n}, w_{1}, w_{2}, \ldots, w_{n}\right\} \cup\left\{q_{1}, q_{2}, \ldots, q_{r}\right\}$ are disjoint,

- $p_{1} p_{2} \cdots p_{2 n}, q_{1} q_{2} \cdots q_{r}$ are induced paths, and $p_{i}$ is not adjacent to $q_{j}$,

- $\left\{v_{1}, \ldots, v_{n}, w_{1}, \ldots, w_{n}\right\}$ is an independent set,

- $v_{i}$ is adjacent to $p_{j}$ if and only if $j=2 i-1$,

- $w_{i}$ is adjacent to $p_{j}$ if and only if $j=2 i$, 
- there exists a sequence $1 \leqslant b_{1}<b_{2}<\cdots<b_{n}<b_{n+1}=r+1$ such that for each $i \in\{1, \ldots, n\}$, $v_{i}$ is adjacent to $q_{b_{i}}$ and non-adjacent to $q_{x}$ for all $x \in\{1, \ldots, r\} \backslash\left\{b_{i}, b_{i}+1, b_{i}+2, \ldots, b_{i+1}-1\right\}$,

- every $w_{i}$ has a neighbor in $S$, and has at most $t-1$ neighbors on $\left\{q_{1}, q_{2}, \ldots, q_{r}\right\}$,

- there are no edges between $S$ and $\left\{p_{1}, p_{2}, \ldots, p_{2 n}, v_{1}, v_{2}, \ldots, v_{n}\right\} \cup\left\{q_{1}, q_{2}, \ldots, q_{r}\right\}$,

- there exists a vertex $w \in S$ where for every $v \in N_{G}\left(\left\{u_{1}, u_{2}, \ldots, u_{n}\right\}\right) \cap S$, there is a path $P$ from $v$ to $w$ in $G[S]$ with $N_{G}\left(\left\{u_{1}, u_{2}, \ldots, u_{n}\right\}\right) \cap V(P)=\{v\}$.

A simple extended hanging ladder is a $t$-extended hanging ladder for some $t \geqslant 2$ such that

- $r=2 n$,

- $v_{i}$ is adjacent to $q_{j}$ if and only if $j=2 i-1$,

- $w_{i}$ is adjacent to $q_{j}$ if and only if $j=2 i$.

Note that the value $t$ is not important in a simple extended hanging ladder because every $w_{i}$ has exactly one neighbor on $\left\{q_{1}, q_{2}, \ldots, q_{r}\right\}$. We depict a simple hanging ladder in Figure 8 .

Lemma 6.12. For an integer $n \geqslant 3$, a simple extended hanging ladder of order $\mu(2 n+2)$ contains $W_{4 n}$ as a vertex-minor.

Proof. Let $G$ be a simple extended hanging ladder of order $\mu(2 n+2)$, and let $A:=V(G) \backslash(S \cup$ $\left.\left\{w_{1}, w_{2}, \ldots, w_{\mu(2 n+2)}\right\}\right)$. By Lemma 6.4, there exist $U \subseteq\left\{w_{1}, w_{2}, \ldots, w_{\mu(2 n+2)}\right\}$ with $|U| \geqslant 2 n+2$ and $v \in S$ and a graph $G^{\prime}$ on $A \cup U \cup\{v\}$ such that

- $G^{\prime}[A \cup U]=G[A \cup U]$,

- $v$ is adjacent to all vertices in $U$ and has no neighbors in $A$ in $G^{\prime}$,

- $G^{\prime}$ is a vertex-minor of $G$.

Let $U:=\left\{w_{i_{1}}, w_{i_{2}}, \ldots, w_{i_{2 n+2}}\right\}$ where $i_{1}<i_{2}<\cdots<i_{2 n+2}$. Then

$$
G_{1} * w_{i_{1}} * w_{i_{2}} * \cdots * w_{i_{2 n+1}} * v_{i_{1}} * v_{i_{2}} * v_{i_{4}} * v_{i_{6}} * \cdots * v_{i_{2 n+2}}
$$

contains an induced subgraph isomorphic to a subdivision of a hanging ladder on $6 n+5$ vertices, and by Lemma 6.8, it contains a vertex-minor isomorphic to $W_{4 n}$.

Lemma 6.13. For every integer $n \geqslant 3$, there exists an integer $L=L(n)$ such that an $n$-extended hanging ladder of order $L$ contains $W_{n}$ as a vertex-minor.

Proof. Let

- $m_{1}:=\mu(n)$,

- $m_{2}:=8 n$,

- $m_{3}:=\mu(2 n+2)$,

- $m_{4}:=m_{1}+2\left(m_{2}-1\right)(n-2)+4$, and 

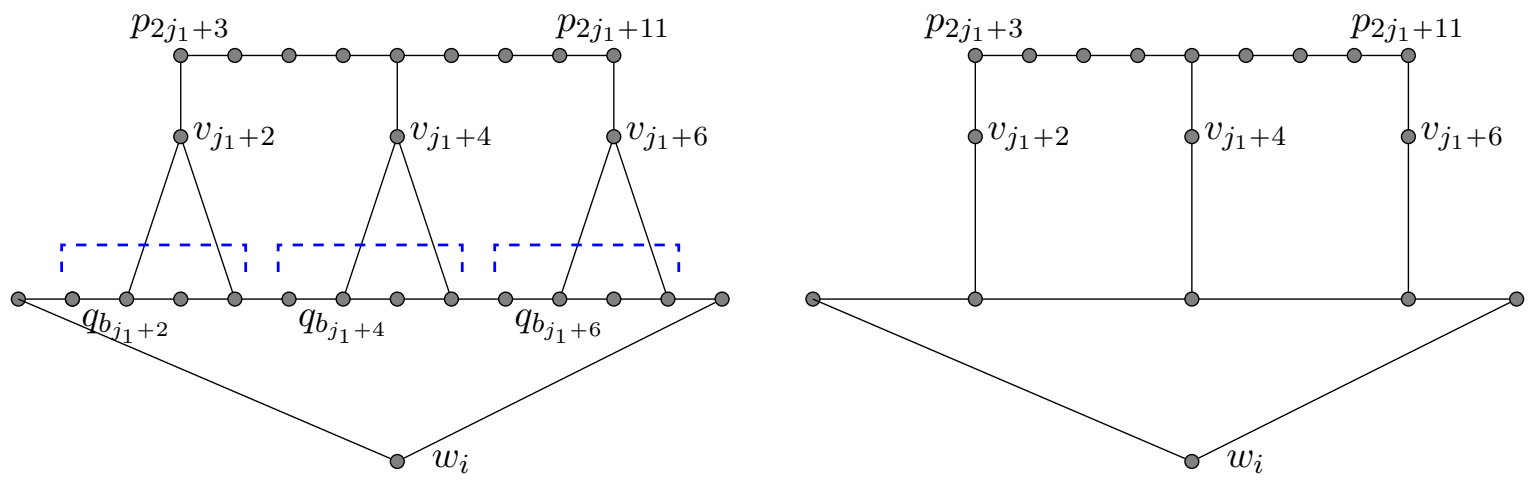

Figure 9: An example of contractions in Claim 6.15 of Lemma 6.13. We will contract dashed parts in the first figure to obtain the second figure.

- $L:=\left(m_{3}-1\right) m_{4}+\frac{m_{4}+m_{1}}{2}$.

Let $G$ be an $n$-extended hanging ladder of order $L$. We claim that $G$ contains $W_{n}$ as a vertex-minor. We first prove two special cases.

Claim 6.14. Suppose there exists $i \in\left\{0,1, \ldots, L-m_{1}\right\}$ such that there are no edges between $\left\{w_{i+1}, w_{i+2}, \ldots, w_{i+m_{1}}\right\}$ and $\left\{q_{b_{i+1}}, q_{b_{i+1}+1}, q_{b_{i+1}+2}, \ldots, q_{b_{i+m_{1}+1}}\right\}$. Then $G$ contains a vertex-minor isomorphic to $W_{n}$.

Proof. Suppose there exists such an integer $i$. Let $i^{\prime}$ be the maximum integer such that $v_{i+1}$ is adjacent to $q_{i^{\prime}}$. Note that $b_{i+1} \leqslant i^{\prime}<b_{i+2}$. Then

$$
q_{i^{\prime}} q_{i^{\prime}+1} \cdots q_{b_{i+m_{1}+1}} v_{i+m_{1}+1} p_{2\left(i+m_{1}+1\right)-1} p_{2\left(i+m_{1}+1\right)-2} \cdots p_{2 i+1} v_{i+1} q_{i^{\prime}}
$$

is an induced cycle. Since there are no edges between $\left\{w_{i+j}: 1 \leqslant j \leqslant m_{1}\right\}$ and $\left\{q_{x}: b_{i+1} \leqslant x \leqslant\right.$ $\left.b_{i+m_{1}+1}\right\}, G$ contains a subdivision of an extended drum of order $m_{1}=\mu(n)$. By Lemma 6.9, $G$ contains a vertex-minor isomorphic to $W_{n}$.

Claim 6.15. If there are $i, j_{1}, j_{2} \in\{1, \ldots, L\}$ with $j_{2}-j_{1} \geqslant m_{2}$ such that

- $w_{i}$ is adjacent to $q_{x_{1}}$ for some $b_{j_{1}} \leqslant x_{1}<b_{j_{1}+1}$ and adjacent to $q_{x_{2}}$ for some $b_{j_{2}} \leqslant x_{2}<b_{j_{2}+1}$,

- $w_{i}$ is not adjacent to $q_{x}$ for all $x \in\left\{x_{1}+1, x_{1}+2, \ldots, x_{2}-1\right\}$,

then $G$ contains a vertex-minor isomorphic to $W_{n}$.

Proof. Suppose there are such integers $i, j_{1}, j_{2}$. Then $w_{i} q_{x_{1}} q_{x_{1}+1} q_{x_{1}+2} \cdots q_{x_{2}} w_{i}$ is an induced cycle.

First assume that $i \leqslant j_{1}$. Let

$$
G_{1}:=G\left[\left\{w_{i}, q_{x_{1}}, q_{x_{1}+1}, \ldots, q_{x_{2}}\right\} \cup\left\{v_{j_{1}+2}, v_{j_{1}+4}, v_{j_{1}+6}, \ldots, v_{j_{2}^{\prime}}\right\} \cup\left\{p_{2 j_{1}+3}, p_{2 j_{1}+4}, \ldots, p_{2 j_{2}^{\prime}-1}\right\}\right],
$$


where $j_{2}^{\prime}=j_{2}-2$ if $j_{2} \equiv j_{1}(\bmod 2)$ and $j_{2}^{\prime}=j_{2}-1$ otherwise. We will contract paths from $G$ to obtain a drum on $\frac{3}{2}\left(m_{2}-2\right) \geqslant 3(2 n-1)$ vertices. See Figure 9 for an example case. Observe that $p_{2 j_{1}+3} p_{2 j_{1}+4} \cdots p_{2 j_{2}^{\prime}-1}$ is a path such that each vertex of $v_{j_{1}+2}, v_{j_{1}+4}, v_{j_{1}+6}, \ldots, v_{j_{2}^{\prime}}$ has a neighbor on this path.

Let $G_{2}$ be the graph obtained from $G_{1}$ by contracting

$$
\left\{q_{b_{j_{1}+(t-1)}}, q_{b_{j_{1}+(t-1)}+1}, \ldots, q_{b_{j_{1}+(t+1)}-1}\right\}
$$

for each $t \in\left\{2,4,6, \ldots, j_{2}^{\prime}-j_{1}\right\}$. By Lemma 2.1, $G_{2}$ is isomorphic to a vertex-minor of $G_{1}$. Moreover, $G_{2}$ contains a subdivision of a drum on $\frac{3}{2}\left(j_{2}-j_{1}-2\right)=\frac{3}{2}\left(m_{2}-2\right) \geqslant 3(2 n-1)$ vertices, and by Lemma 6.6, $G_{2}$ contains a vertex-minor isomorphic to $W_{n}$. The case when $i \geqslant j_{2}$ is symmetric to the previous case. We may assume that $j_{1}<i<j_{2}$. In this case, $w_{i}$ is adjacent to $p_{2 i}$, and to avoid having this edge, we take a part that is larger and having no edges from $w_{i}$.

Since $j_{2}-j_{1} \geqslant m_{2}$, we have either $i-j_{1} \geqslant m_{2} / 2$ or $j_{2}-i \geqslant m_{2} / 2$. So, by taking the longer path between two subpaths obtained from $p_{2 j_{1}+3} p_{2 j_{1}+4} \cdots p_{2 j_{2}^{\prime}-1}$ by removing $p_{2 i}$, we can observe that $G$ contains a vertex-minor isomorphic to a drum on $\frac{3}{2}\left(\frac{m_{2}}{2}-2\right)$ vertices. Since $\frac{3}{2}\left(\frac{m_{2}}{2}-2\right)=3(2 n-1)$, by Lemma 6.6, $G$ contains a vertex-minor isomorphic to $W_{n}$.

From Claim 6.15, we observe the following.

Claim 6.16. If there are $i, j_{1}, j_{2} \in\{1, \ldots, L\}$ with $j_{2}-j_{1} \geqslant\left(m_{2}-1\right)(n-2)+1$ such that $w_{i}$ is adjacent to $q_{x_{1}}$ for some $b_{j_{1}} \leqslant x_{1}<b_{j_{1}+1}$ and adjacent to $q_{x_{2}}$ for some $b_{j_{2}} \leqslant x_{2}<b_{j_{2}+1}$, then $G$ contains a vertex-minor isomorphic to $W_{n}$.

Proof. Since $j_{2}-j_{1} \geqslant\left(m_{2}-1\right)(n-2)+1$ and $w_{i}$ has at most $n-1$ neighbors in $\left\{q_{1}, q_{2}, \ldots, q_{r}\right\}$, there exist $j_{3}, j_{4}$ with $j_{1}<j_{3}<j_{4}<j_{2}$ such that

- $j_{4}-j_{3} \geqslant m_{2}$,

- $w_{i}$ is adjacent to $q_{x_{3}}$ for some $b_{j_{3}} \leqslant x_{3}<b_{j_{3}+1}$ and adjacent to $q_{x_{4}}$ for some $b_{j_{4}} \leqslant x_{4}<b_{j_{4}+1}$,

- $w_{i}$ is not adjacent to $q_{x}$ for all $x_{3}<x<x_{4}$.

By Claim 6.15, $G$ contains a vertex-minor isomorphic to $W_{n}$.

For each $i \in\left\{1,2, \ldots, m_{3}\right\}$, we choose a vertex $w_{d_{i}}$ such that

- $d_{i} \in\left\{i m_{4}+1, i m_{4}+2, \ldots, i m_{4}+m_{1}\right\}$ and

- $w_{d_{i}}$ is adjacent to $q_{x}$ for some $b_{i m_{4}+1} \leqslant x \leqslant b_{i m_{4}+m_{1}+1}$.

If such a vertex does not exist for some $i$, then by Claim 6.14, $G$ contains a vertex-minor isomorphic to $W_{n}$. We may assume that such a vertex exists for each $i \in\left\{1,2, \ldots, m_{3}\right\}$.

Suppose $w_{d_{i}}$ is adjacent to $q_{y}$ for some $y \geqslant b_{i m_{4}+\frac{m_{4}+m_{1}}{2}}$ or for some $y \leqslant b_{i m_{4}-\frac{m_{4}-m_{1}}{2}+3}-1$. By the choice of $d_{i}, w_{d_{i}}$ is adjacent to $q_{x}$ for some $b_{i m_{4}+1} \leqslant x \leqslant b_{i m_{4}+m_{1}+1}$. Since $\frac{m_{4}+m_{1}^{2}}{2}-\left(m_{1}+1\right) \geqslant$ $\left(m_{1}-1\right)(n-2)+1$ and $1-\left(-\frac{m_{4}-m_{1}}{2}+2\right) \geqslant\left(m_{1}-1\right)(n-2)+1$, by Claim 6.16, $G$ contains a vertex-minor isomorphic to $W_{n}$. We may assume that each $w_{d_{i}}$ is not adjacent to $q_{y}$ for all $y \in$

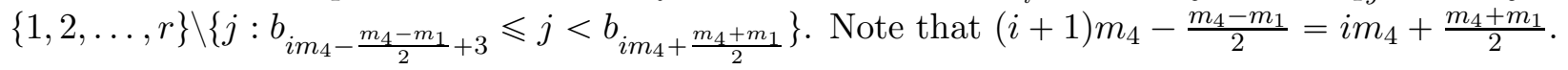


Let $G_{1}$ be the subgraph of $G$ induced on

$$
\left\{w_{d_{i}}: 1 \leqslant i \leqslant m_{3}\right\} \cup\left\{v_{i m_{4}-\frac{m_{4}-m_{1}}{2}+1}: 1 \leqslant i \leqslant m_{3}\right\} \cup\left\{p_{1}, \ldots, p_{2 L}, q_{1}, \ldots, q_{r}\right\} \cup S .
$$

Let $G_{2}$ be the graph obtained from $G_{1}$ by contracting

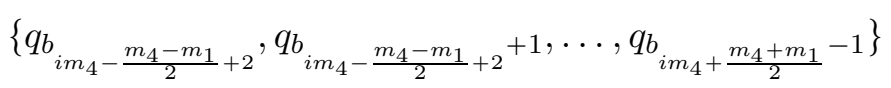

for each $i \in\left\{1,2, \ldots, m_{3}\right\}$ and contracting

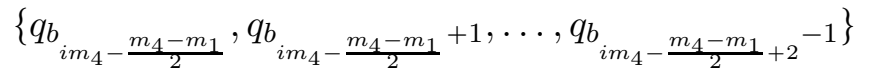

for each $i \in\left\{1,2, \ldots, m_{3}\right\}$. By Lemma 2.1, $G_{2}$ is isomorphic to a vertex-minor of $G$. Also, $G_{2}$ contains a subdivision of a simple extended hanging ladder of order $m_{3}=\mu(2 n+2)$. By Lemma 6.12, $G_{2}$ contains a vertex-minor isomorphic to $W_{4 n}$.

\section{$7 \quad(w, \ell)$-patched cycles}

Let $w, \ell$ be positive integers. A $(w, \ell)$-patched cycle $\left(q_{1} q_{2} \cdots q_{m} q_{1}, S_{1}, S_{2}, \ldots, S_{w}\right)$ is a graph $G$ on pairwise disjoint sets $\left\{q_{1}, q_{2}, \ldots, q_{m}\right\}$ and $S_{i}=\left\{s_{1}^{i}, s_{2}^{i}, \ldots, s_{\ell}^{i}\right\}$ for each $i \in\{1, \ldots, w\}$ satisfying the following.

(1) $q_{1} q_{2} \cdots q_{m} q_{1}$ is an induced cycle.

(2) There exists a sequence $1 \leqslant b_{1}<b_{2}<\cdots<b_{\ell} \leqslant m$ such that for each $i \in\{1, \ldots, w\}$ and $j \in\{1,2, \ldots, \ell\}, s_{j}^{i}$ is adjacent to $q_{b_{j}}$ and non-adjacent to $q_{b_{x}}$ for all $x \in\{1, \ldots, \ell\} \backslash\{1, \ldots, j\}$.

We call $w$ and $\ell$ the width and length respectively, of a $(w, \ell)$-patched cycle. Note that $m \geqslant \ell$. A $(w, \ell)$-patched cycle $\left(q_{1} q_{2} \cdots q_{m} q_{1}, S_{1}, S_{2}, \ldots, S_{w}\right)$ is simple if $S_{1} \cup S_{2} \cup \cdots \cup S_{w}$ is an independent set.

In Subsection 7.1, we show that for every $n$, there exists $M$ such that every graph $G$ obtained from a simple $(2, M)$-patched cycle $\left(q_{1} q_{2} \cdots q_{m} q_{1}, S_{1}, S_{2}\right)$ by adding two disjoint vertex sets $T_{1}$ and $T_{2}$ such that

- there are no edges between $\left\{q_{1}, q_{2}, \ldots, q_{m}\right\}$ and $T_{1} \cup T_{2}$,

- there are no edges between $S_{1}$ and $T_{2}$,

- for each $i \in\{1,2\}$, every vertex in $S_{i}$ has a neighbor in $T_{i}$,

- for each $i \in\{1,2\}$, there exists a vertex $r_{i} \in T_{i}$ where for every $v \in N_{G}\left(S_{i}\right) \cap T_{i}$, there is a path $P$ from $v$ to $r_{i}$ in $G\left[T_{i}\right]$ with $N_{G}\left(S_{i}\right) \cap V(P)=\{v\}$,

contains a vertex-minor isomorphic to $W_{n}$. This is the motivation for introducing $(w, \ell)$-patched cycles. In Subsection [7.2, we show that for every $n$, a simple $(2, n)$-patched cycle can be obtained from a patched cycle with sufficiently large width and large length using the rectangular Ramsey lemma developed in Section 5. In Subsection 7.3, we discuss how to obtain a huge patched cycle from a structure that can be naturally extracted from a graph with bounded clique number and sufficiently large chromatic number. 


\subsection{From a simple $(2, M)$-patched cycle with attached connected subgraphs}

Proposition 7.1. For every integer $n \geqslant 3$, there exists an integer $M=M(n)$ satisfying the following property: If $G$ is the graph obtained from a simple $(2, M)$-patched cycle $\left(q_{1} q_{2} \cdots q_{m} q_{1}, S_{1}, S_{2}\right)$ by adding disjoint vertex sets $T_{1}$ and $T_{2}$ such that

- there are no edges between $\left\{q_{1}, q_{2}, \ldots, q_{m}\right\}$ and $T_{1} \cup T_{2}$,

- there are no edges between $S_{1}$ and $T_{2}$,

- for each $i \in\{1,2\}$, every vertex in $S_{i}$ has a neighbor in $T_{i}$,

- for each $i \in\{1,2\}$, there exists a vertex $r_{i} \in T_{i}$ where for every $v \in N_{G}\left(S_{i}\right) \cap T_{i}$, there is a path $P$ from $v$ to $r_{i}$ in $G\left[T_{i}\right]$ with $N_{G}\left(S_{i}\right) \cap V(P)=\{v\}$,

then $G$ contains a vertex-minor isomorphic to $W_{n}$.

Proof. We recall that $\mu(n)=(n-1)\left(R(n, n)^{2 n-3}+1\right)$ for $n \geqslant 3, N^{\prime}$ is the function defined in Corollary 4.3, and $L$ is the function defined in Lemma 6.13. We note that $L(n) \geqslant \mu(n)+\mu(2 n+1)-1$. Let

- $M_{1}:=(n-1)(4 L(n)+6)$,

- $M_{2}:=(n-1) N^{\prime}\left(n-1, M_{1}\right)$,

- $M:=N^{\prime}\left(n-1, M_{2}\right)$.

For each $i \in\{1,2\}$, let $S_{i}:=\left\{s_{1}^{i}, \ldots, s_{M}^{i}\right\}$, and let $b_{1}, \ldots, b_{M}$ be a sequence such that

- $1 \leqslant b_{1}<b_{2}<\cdots<b_{M} \leqslant m$ and

- for each $i \in\{1,2\}$ and $j \in\{1,2, \ldots, M\}, s_{j}^{i}$ is adjacent to $q_{b_{j}}$ and non-adjacent to $q_{b_{x}}$ for all $x \in\{1, \ldots, M\} \backslash\{1, \ldots, j\}$.

Such a sequence exists by the definition of a $(2, M)$-patched cycle. Let $Q:=\left\{q_{1}, q_{2}, \ldots, q_{m}\right\}$, and for each $i \in\{1,2\}$ and $j \in\{1, \ldots, M\}$, let $N_{j}^{i}=\left\{k: q_{k} \in N_{G}\left(s_{j}^{i}\right), 1 \leqslant k \leqslant M\right\}$.

Note that $m \geqslant M \geqslant n+3$. If there is a vertex in $S_{1} \cup S_{2}$ having at least $n$ neighbors on $Q$, then $G$ contains a vertex-minor isomorphic to $W_{n}$ by Lemma 6.5. We may assume that each vertex in $S_{1} \cup S_{2}$ has at most $n-1$ neighbors in $Q$. In other words, for each $i \in\{1,2\}$ and $j \in\{1, \ldots, M\}$, $\left|N_{j}^{i}\right| \leqslant n-1$.

We apply Corollary 4.3 to $\left(N_{1}^{1}, \ldots, N_{M}^{1}\right)$. Then there exist a sequence $1 \leqslant c_{1}<c_{2}<\cdots<$ $c_{M_{2}} \leqslant M$ and a regular partition $\mathcal{I}_{1}$ of $\mathbb{R}$ with respect to $\left(N_{c_{1}}^{1}, N_{c_{2}}^{1}, \ldots, N_{c_{M_{2}}}^{1}\right)$ such that $\mathcal{I}_{1}$ has order at most $n-1$. Since $\mathcal{I}_{1}$ has order at most $n-1$, there exists a part $I_{1}$ of $\mathcal{I}_{1}$ that contains at least $\frac{M_{2}}{n-1}$ integers in $\left\{b_{c_{1}}, b_{c_{2}}, \ldots, b_{c_{M_{2}}}\right\}$. Let $x$ be the minimum such that $x \geqslant 1$ and $b_{c_{x}} \in I_{1}$, and let $y$ be the maximum such that $y \leqslant M_{2}$ and $b_{c_{y}} \in I_{1}$. Then $y-x+1 \geqslant \frac{M_{2}}{n-1}=N^{\prime}\left(n-1, M_{1}\right)$.

We apply Corollary 4.3 again to $\left(N_{1}^{2} \cap I_{1}, \ldots, N_{M}^{2} \cap I_{1}\right)$. Then there exist a subsequence $d_{1}<d_{2}<\cdots<d_{M_{1}}$ of $c_{x}, c_{x+1}, \ldots, c_{y}$ and a regular partition $\mathcal{I}_{2}$ of $I_{1}$ with respect to $\left(N_{d_{1}}^{2} \cap\right.$ $\left.I_{1}, N_{d_{2}}^{2} \cap I_{1}, \ldots, N_{d_{M_{1}}}^{2} \cap I_{1}\right)$ such that $\mathcal{I}_{2}$ has order at most $n-1$. There exists a part $I_{2}$ of $\mathcal{I}_{2}$ that contains at least $\frac{M_{1}}{n-1}$ integers in $\left\{b_{d_{1}}, b_{d_{2}}, b_{d_{3}}, \ldots, b_{d_{M_{1}}}\right\}$. Let $x^{\prime}$ be the minimum such that $x^{\prime} \geqslant 1$ 
and $b_{d_{x^{\prime}}} \in I_{2}$ and let $y^{\prime}$ be the maximum such that $y^{\prime} \leqslant M_{1}$ and $b_{d_{y^{\prime}}} \in I_{2}$. Let $a=y^{\prime}-x^{\prime}+1$. Then $a=y^{\prime}-x^{\prime}+1 \geqslant \frac{M_{1}}{n-1}=4 L(n)+6$. Let $u_{1}=d_{x^{\prime}}, u_{2}=d_{x^{\prime}+1}, \ldots, u_{a}=d_{y^{\prime}}$.

By the definition of a $(2, M)$-patched cycle, for each $i \in\{1,2\}$ and $1 \leqslant j \leqslant a, s_{u_{j}}^{i}$ is adjacent to $q_{b_{u_{j}}}$ but non-adjacent to vertices in $\left\{q_{b_{u_{j+1}}}, q_{b_{u_{j+2}}}, \ldots, q_{b_{u_{a}}}\right\}$. Therefore, $N_{u_{j}}^{i} \cap I_{2}$ is not identical for $1 \leqslant j \leqslant a$ and moreover, minimal intervals containing $N_{u_{1}}^{i} \cap I_{2}, N_{u_{2}}^{i} \cap I_{2}, \ldots, N_{u_{a}}^{i} \cap I_{2}$ appear in the same order as $u_{1}, u_{2}, \ldots, u_{a}$. In other words, for each $i \in\{1,2\}$,

- $N_{u_{1}}^{i} \subseteq\left(-\infty, b_{u_{2}}\right) \cap I_{2}$,

- $N_{u_{a}}^{i} \subseteq\left(b_{u_{a-1}}, \infty\right) \cap I_{2}$,

- for $j \in\{2,3, \ldots, a-1\}, N_{u_{j}}^{i} \subseteq\left(b_{u_{j-1}}, b_{u_{j+1}}\right)$.

Let $t=4 L(n)+4$.

We first deal with the case when $\mathcal{I}_{1}$ consists of one part.

Claim 7.2. If $\mathcal{I}_{1}$ consists of one part, then $G$ contains a vertex-minor isomorphic to $W_{n}$.

Proof. Let $G_{1}$ be the subgraph of $G$ induced on $Q \cup\left\{s_{u_{2}}^{1}, s_{u_{4}}^{1}, s_{u_{6}}^{1}, \ldots, s_{u_{t}}^{1}\right\} \cup T_{1}$. We obtain a graph $G_{2}$ from $G_{1}$ by contracting $\left\{q_{x}: b_{u_{j-1}} \leqslant x<b_{u_{j+1}}\right\}$ for each $j \in\{2,4, \ldots, t\}$ to a vertex. By Lemma 2.1, $G_{2}$ is isomorphic to a vertex-minor of $G_{1}$. Then $G_{2}$ is a subdivision of an extended drum of order $t / 2 \geqslant L(n) \geqslant \mu(n)$. By Lemma 6.9, $G$ contains a vertex-minor isomorphic to $W_{n}$.

By Claim 7.2, we may assume that $\mathcal{I}_{1}$ consists of at least two parts. Let $J$ be a part of $\mathcal{I}_{1}$ other than $I_{1}$. Clearly, $J$ is disjoint from $I_{2}$. By the definition of a regular partition, either

(1) $N_{u_{2}}^{1} \cap J=N_{u_{4}}^{1} \cap J=\cdots=N_{u_{t}}^{1} \cap J \neq \varnothing$, or

(2) $\left|N_{u_{2}}^{1} \cap J\right|=\left|N_{u_{4}}^{1} \cap J\right|=\cdots=\left|N_{u_{t}}^{1} \cap J\right|>0$ and for all $i, j \in\{2,4, \ldots, t\}$ with $i<j$, $\max \left(N_{u_{i}}^{1} \cap J\right)<\min \left(N_{u_{j}}^{1} \cap J\right)$, or

(3) $\left|N_{u_{2}}^{1} \cap J\right|=\left|N_{u_{4}}^{1} \cap J\right|=\cdots=\left|N_{u_{t}}^{1} \cap J\right|>0$ and for all $i, j \in\{2,4, \ldots, t\}$ with $i<j$, $\max \left(N_{u_{j}}^{1} \cap J\right)<\min \left(N_{u_{i}}^{1} \cap J\right)$.

When (1) appears, we will find an extended clam of large order, and when (2) or (3) appears, we will find an extended hanging ladder of large order.

Case 1. $N_{u_{2}}^{1} \cap J=N_{u_{4}}^{1} \cap J=\cdots=N_{u_{t}}^{1} \cap J \neq \varnothing$.

Let $w \in N_{u_{2}}^{1} \cap J$. Let $Q_{1}$ be the connected component of $G[Q]-q_{b_{u_{1}}}-q_{b_{u_{a}}}$ containing $q_{b_{u_{2}}}$. We observe that $q_{w} \notin V\left(Q_{1}\right)$ and $q_{b_{u_{4}}}, q_{b_{u_{6}}}, \ldots, q_{b_{u_{t-2}}} \in V\left(Q_{1}\right)$. Let $G_{1}$ be the subgraph of $G$ induced on

$$
\begin{aligned}
\left\{q_{w}\right\} & \cup V\left(Q_{1}\right) \cup T_{2} \\
& \cup\left\{s_{u_{j}}^{1}: 4 \leqslant j \leqslant t-2, j \equiv 0 \quad(\bmod 4)\right\} \cup\left\{s_{u_{j}}^{2}: 4 \leqslant j \leqslant t-2, j \equiv 2 \quad(\bmod 4)\right\} .
\end{aligned}
$$

We obtain a graph $G_{2}$ from $G_{1}$ by contracting $\left\{q_{x}: b_{u_{j-1}} \leqslant x<b_{u_{j+1}}\right\}$ for each $j \in\{4,6,8, \ldots, t-2\}$ to a vertex. By Lemma 2.1, $G_{2}$ is isomorphic to a vertex-minor of $G_{1}$. Note that there are no edges between $T_{2}$ and $S_{1}$. Thus, $G_{2}$ contains a subdivision of an extended clam of order 
$t / 4-1=L(n) \geqslant \mu(n)+\mu(2 n+1)-1$, and by Lemma 6.11, $G$ contains a vertex-minor isomorphic to $W_{n}$.

Case 2. $\left|N_{u_{2}}^{1} \cap J\right|=\left|N_{u_{4}}^{1} \cap J\right|=\cdots=\left|N_{u_{t}}^{1} \cap J\right|>0$ and for all $i, j \in\{2,4, \ldots, t\}$ with $i<j$, $\max \left(N_{u_{i}}^{1} \cap J\right)<\min \left(N_{u_{j}}^{1} \cap J\right)$.

Let $Q_{1}$ be the connected component of $G[Q]-q_{b_{u_{1}}}-q_{b_{u_{a}}}$ containing $q_{b_{u_{2}}}$. Let $Q_{2}$ be the path on $\left\{q_{i}: i \in J\right\}$. Note that there are no edges between $Q_{1}$ and $Q_{2}$. Let $G_{1}$ be the subgraph of $G$ induced on

$$
\begin{aligned}
V\left(Q_{1}\right) & \cup V\left(Q_{2}\right) \cup T_{2} \\
& \cup\left\{s_{u_{j}}^{1}: 4 \leqslant j \leqslant t-2, j \equiv 0 \quad(\bmod 4)\right\} \cup\left\{s_{u_{j}}^{2}: 4 \leqslant j \leqslant t-2, j \equiv 2 \quad(\bmod 4)\right\} .
\end{aligned}
$$

We obtain a graph $G_{2}$ from $G_{1}$ by contracting $\left\{q_{x}: b_{u_{j-1}} \leqslant x<b_{u_{j+1}}\right\}$ for each $j \in\{4,6,8, \ldots, t-2\}$. By Lemma 2.1. $G_{2}$ is isomorphic to a vertex-minor of $G_{1}$. Note that there are no edges between $T_{2}$ and $S_{1}$. Thus, $G_{2}$ contains a subdivision of an $n$-extended hanging ladder of order $t / 4-1 \geqslant L(n)$, and by Lemma 6.13, $G$ contains a vertex-minor isomorphic to $W_{n}$.

Case 3. For all $i, j \in\{2,4, \ldots, t\}$ with $i<j,\left|N_{u_{i}}^{1} \cap J\right|=\left|N_{u_{j}}^{1} \cap J\right|>0$ and $\max \left(N_{u_{j}}^{1} \cap J\right)<$ $\min \left(N_{u_{i}}^{1} \cap J\right)$.

This case is symmetric to Case 2.

This completes the proof of the proposition.

\subsection{Obtaining a simple patched cycle}

Proposition 7.3. Let $R_{1}, R_{2}$ be the functions defined in Proposition 5.2. For all positive integers $a, b$, and $k$, if $M=R_{1}(a, b, k)$ and $N=R_{2}(a, b, k)$, then every $(M, N)$-patched cycle $\left(q_{1} q_{2} \cdots q_{m} q_{1}, S_{1}, S_{2}, \ldots, S_{M}\right)$ contains either a clique of size $k$ or a simple $(a, b)$-patched cycle $\left(q_{1} q_{2} \cdots q_{m} q_{1}, T_{1}, T_{2}, \ldots, T_{a}\right)$ where $T_{1}, \ldots, T_{a}$ are contained in pairwise distinct sets of $S_{1}, \ldots, S_{M}$.

Proof. For each $i \in\{1, \ldots, M\}$, let $S_{i}:=\left\{s_{1}^{i}, s_{2}^{i}, \ldots, s_{N}^{i}\right\}$ and let $1 \leqslant b_{1}<b_{2}<\cdots<b_{N} \leqslant m$ be a sequence such that

- for each $i \in\{1, \ldots, M\}$ and each $j \in\{1, \ldots, N\}, s_{j}^{i}$ is adjacent to $q_{b_{j}}$ and non-adjacent to $q_{b_{x}}$ for all $x>j$.

By Proposition [5.2, either $G$ has a clique of $k$ vertices or there exist $X \subseteq\{1,2, \ldots, M\}$ and $Y \subseteq\{1,2, \ldots, N\}$ such that $\left\{s_{j}^{i}: i \in X, j \in Y\right\}$ is an independent set and $|X|=a,|Y|=b$. In the latter case, let $X=\left\{x_{1}, x_{2}, \ldots, x_{a}\right\}$ and $T_{i}:=\left\{s_{j}^{x_{i}}: j \in Y\right\}$ for each $i=1,2, \ldots, a$. It is easy to verify that $\left(q_{1} q_{2} \cdots q_{m} q_{1}, T_{1}, T_{2}, \ldots, T_{a}\right)$ is a simple $(a, b)$-patched cycle.

\subsection{Obtaining a patched cycle with large width and length}

We prove the following.

Proposition 7.4. Let $k>0, \ell>0, n \geqslant 2$ be integers and let $M:=\ell n^{k}$. Let $G$ be a graph on the vertex set $\left\{q_{1}, q_{2}, \ldots, q_{M}\right\} \cup V_{1} \cup V_{2} \cup \ldots \cup V_{k}$ such that

- $\left\{q_{1}, q_{2}, \ldots, q_{M}\right\}, V_{1}, V_{2}, \ldots, V_{k}$ are pairwise disjoint, 
- $q_{1} q_{2} q_{3} \cdots q_{M} q_{1}$ is an induced cycle,

- for each $i \in\{1,2, \ldots, M\}$ and each $j \in\{1, \ldots, k\}$, q $q_{i}$ has a neighbor in $V_{j}$,

- for each vertex $v \in V(G) \backslash\left\{q_{1}, q_{2}, \ldots, q_{M}\right\}, v$ has at most $n-1$ neighbors in $\left\{q_{1}, q_{2}, \ldots, q_{M}\right\}$.

Then $G$ contains a $(k, \ell)$-patched cycle $\left(q_{1} q_{2} \cdots q_{M} q_{1}, S_{1}, S_{2}, \ldots, S_{k}\right)$ such that $S_{i} \subseteq V_{i}$ for each $i \in\{1, \ldots, k\}$.

Proof. We prove the statement by induction on $k$.

First assume that $k=1$. Let $s_{1}$ be a neighbor of $q_{1}$ in $V_{1}$, and let $b_{1}:=1$. Let $i$ be the maximum integer satisfying the following: there exist distinct vertices $s_{1}, s_{2}, \ldots, s_{i}$ of $V_{1}$ and a sequence $b_{1}<b_{2}<\cdots<b_{i}$ where for all $x \in\{1, \ldots, i\}, s_{x}$ is adjacent to $q_{b_{x}}$ and when $x>1$,

- $b_{x}$ is the minimum integer such that $b_{x}>b_{x-1}$ and $q_{b_{x}}$ has no neighbors in $\left\{s_{1}, \ldots, s_{x-1}\right\}$.

Such $i$ exists, because $i=1$ satisfies the conditions. Suppose that $i<\ell$. Note that every vertex $q_{j}$ for $1 \leqslant j \leqslant b_{i}$ has a neighbor in $\left\{s_{1}, \ldots, s_{i}\right\}$, otherwise, let $j^{\prime}$ be the smallest integer such that $b_{j^{\prime}}>j$ and we may replace $b_{j^{\prime}}$ with $j$, contradicting our assumption on $b_{j^{\prime}}$. Therefore, vertices in $\left\{s_{1}, \ldots, s_{i}\right\}$ may have at most $(n-1) i-b_{i}$ neighbors $q_{j}$ for $j>b_{i}$. It implies that there exists $j$ with $b_{i}<j \leqslant(n-1) i+1 \leqslant \ell n$ such that $q_{j}$ has no neighbors in $\left\{s_{1}, \ldots, s_{i}\right\}$. So, we can extend the sequence by taking $b_{i+1}:=j$ and a neighbor of $q_{b_{i+1}}$ in $V_{1}$ as $s_{i+1}$, contradicting to the maximality of $i$. Thus, we have $i \geqslant \ell$. Note that by the choice of $b_{1}, \ldots, b_{i}$, this sequence satisfies the property that

- for each $x \in\{1, \ldots, i\}, s_{x}$ is adjacent to $q_{b_{x}}$ and non-adjacent to $q_{b_{y}}$ for all $y>x$.

We conclude $G$ contains a $(1, \ell)$-patched cycle $\left(q_{1} q_{2} \cdots q_{M} q_{1}, S_{1}\right)$ with $S_{1} \subseteq V_{1}$.

Now, suppose $k>1$. By the induction hypothesis, $G$ contains a $(k-1, \ell n)$-patched cycle $\left(q_{1} q_{2} \cdots q_{M} q_{1}, T_{1}, \ldots, T_{k-1}\right)$ such that $T_{i} \subseteq V_{i}$ for each $i \in\{1, \ldots, k-1\}$. Let $T_{i}=\left\{t_{1}^{i}, t_{2}^{i}, \ldots, t_{\ell n}^{i}\right\}$ for each $i \in\{1, \ldots, k-1\}$ and let $1 \leqslant b_{1}<b_{2}<\cdots<b_{\ell n} \leqslant M$ be the sequence such that

- for each $i \in\{1, \ldots, k-1\}$ and $j \in\{1,2, \ldots, \ell n\}, t_{j}^{i}$ is adjacent to $q_{b_{j}}$ and non-adjacent to $q_{b_{x}}$ for all $x \in\{j+1, \ldots, \ell n\}$.

For each $i \in\{1, \ldots, k-1\}$, let $f_{i}:\left\{b_{1}, \ldots, b_{\ell(n-1)+1}\right\} \rightarrow T_{i}$ be the bijection such that $f_{i}\left(b_{j}\right)=t_{j}^{i}$.

Let $s_{1}^{k} \in V_{k}$ be a neighbor of $q_{b_{1}}$, and let $c_{1}:=1$. Let $i$ be the maximum integer satisfying the following: there exist distinct vertices $s_{1}^{k}, s_{2}^{k}, \ldots, s_{i}^{k}$ of $V_{k}$ and a sequence $c_{1}<c_{2}<\cdots<c_{i}$ where for all $x \in\{1, \ldots, i\}, q_{b_{c_{x}}}$ is adjacent to $s_{x}^{k}$, and when $x>1$,

- $c_{x}$ is the minimum integer such that $c_{x}>c_{x-1}$ and $q_{b_{c_{x}}}$ has no neighbors in $\left\{s_{1}^{k}, \ldots, s_{x-1}^{k}\right\}$,

Such $i$ exists, because $i=1$ satisfies the conditions. Suppose that $i<\ell$. Note that every vertex $q_{b_{j}}$ in $\left\{q_{b_{1}}, q_{b_{2}}, \ldots, q_{b_{c_{i}}}\right\}$ has a neighbor in $\left\{s_{1}^{k}, \ldots, s_{i}^{k}\right\}$, otherwise, let $j^{\prime}$ be the smallest integer such that $c_{j^{\prime}}>j$ and we may replace $c_{j^{\prime}}$ by $j$, contradicting our assumption on $c_{j^{\prime}}$. Therefore, vertices in $\left\{s_{1}^{k}, \ldots, s_{i}^{k}\right\}$ may have at most $(n-1) i-c_{i}$ neighbors in $\left\{q_{b_{j}}: c_{i}<j \leqslant \ell n\right\}$. It implies that there exists $j$ with $c_{i}<j \leqslant(n-1) i+1 \leqslant \ell n$ such that $q_{b_{j}}$ has no neighbors in $\left\{s_{1}^{k}, \ldots, s_{i}^{k}\right\}$. So, we can extend the sequence by taking $c_{i+1}:=j$ and a neighbor of $q_{c_{i+1}}$ in $V_{k}$ as $s_{i+1}^{k}$, contradicting to the maximality of $i$. Thus, we have $i \geqslant \ell$. Note that by the choice of $c_{1}, \ldots, c_{i}$, this sequence satisfies the property that 
- for each $x \in\{1, \ldots, i\}, s_{x}^{k}$ is adjacent to $q_{b_{c_{x}}}$ and non-adjacent to $q_{b_{c y}}$ for all $y>x$.

For each $i \in\{1, \ldots, k-1\}$ and $j \in\{1, \ldots, \ell\}$, let $s_{j}^{i}$ be the vertex $f_{i}\left(c_{j}\right)$. Then

$$
\left(q_{1} q_{2} \cdots q_{M} q_{1},\left\{s_{1}^{1}, \ldots, s_{\ell}^{1}\right\}, \ldots,\left\{s_{1}^{k}, \ldots, s_{\ell}^{k}\right\}\right)
$$

is a $(k, \ell)$-patched cycle such that $\left\{s_{1}^{i}, \ldots, s_{\ell}^{i}\right\} \subseteq V_{i}$ for each $i \in\{1, \ldots, k\}$.

\section{Main theorem}

We use the following theorem.

Theorem 8.1 (Chudnovsky, Scott, and Seymour [8]). For every integer $n \geqslant 3$, the class of graphs having no induced cycle of length at least $n$ is $\chi$-bounded.

Theorem 8.2. For every integer $n \geqslant 3$, the class of graphs with no $W_{n}$ vertex-minor is $\chi$-bounded.

Proof. We recall that $R_{1}, R_{2}$ are the functions defined in Proposition [5.2, and $M$ is the function defined in Proposition 7.1. Let $g_{k}$ be the $\chi$-bounding function of Theorem 8.1 such that for every graph $G$ having no induced cycle of length at least $k$ and all induced subgraphs $H$ of $G, \chi(H) \leqslant$ $g_{k}(\omega(H))$.

Let $G$ be a graph such that $\omega(G) \leqslant q$ for some positive integer $q$ and it has no vertex-minor isomorphic to $W_{n}$. Let $R_{1}:=R_{1}(2, M(n), q+1), R_{2}:=R_{2}(2, M(n), q+1)$, and $r:=R_{2} n^{R_{1}}$. We claim that $\chi(G) \leqslant g_{r}(q) \cdot 2^{R_{1}}$. Suppose not. We may assume that $G$ is connected as we can color each connected component separately.

We will find a simple $(2, M(n))$-patched cycle with additional vertex sets described in Proposition 7.1.

Let $v_{1}$ be a vertex of $G$ and for $i \geqslant 0$, let $L_{i}^{1}$ be the set of all vertices of $G$ whose distance to $v_{1}$ is $i$ in $G$. If each $L_{j}^{1}$ is $g_{r}(q) \cdot 2^{R_{1}-1}$-colorable, then $G$ is $g_{r}(q) \cdot 2^{R_{1}}$-colorable. Therefore there exists

a level $L_{t}^{1}$ such that $\chi\left(G\left[L_{t}^{1}\right]\right)>g_{r}(q) \cdot 2^{R_{1}-1} \geqslant g_{r}(q)$. Thus $G\left[L_{t}^{1}\right]$ contains an induced cycle of length at least $r$ by Theorem 8.1. Since $r \geqslant n+3$ and $G$ has no vertex-minor isomorphic to $W_{n}$, by Lemma 6.5, we have $t \geqslant 2$. Let $X_{1}:=L_{t}^{1}, Y_{1}:=L_{t-1}^{1}, Z_{1}:=L_{0}^{1} \cup L_{1}^{1} \cup \cdots \cup L_{t-2}^{1}$, and $r_{1}$ be the vertex in $L_{0}^{1}$. We note that for every $v \in N_{G}\left(Y_{1}\right) \cap Z_{1}$, there is a path $P=p_{0} p_{1} p_{2} \cdots p_{t-2}$ where $p_{0}=r_{1}$, $p_{t-2}=v$ and for each $i \in\{0, \ldots, t-2\}, p_{i} \in L_{i}^{1}$. This path satisfies that $N_{G}\left(Y_{1}\right) \cap V(P)=\{v\}$.

Let $i$ be the maximum integer in $\left\{1,2, \ldots, R_{1}\right\}$ such that there exist disjoint vertex sets $X_{i}$ and $Y_{1}, \ldots, Y_{i}$ and $Z_{1}, \ldots, Z_{i}$ such that

- $\chi\left(G\left[X_{i}\right]\right)>g_{r}(q) \cdot 2^{R_{1}-i}$,

- for each vertex $v \in X_{i}$ and each $x \in\{1, \ldots, i\}, v$ has a neighbor in $Y_{x}$ and no neighbors in $Z_{x}$,

- for each $x \in\{1, \ldots, i\}$, every vertex in $Y_{x}$ has a neighbor in $Z_{x}$,

- for each $x \in\{1, \ldots, i\}$, there exists a vertex $r_{x} \in Z_{x}$ where for every $v \in N_{G}\left(Y_{x}\right) \cap Z_{x}$, there is a path $P$ from $v$ to $r_{x}$ in $G\left[Z_{x}\right]$ with $N_{G}\left(Y_{x}\right) \cap V(P)=\{v\}$,

- for distinct integers $x, y \in\{1, \ldots, i\}$ with $x<y$, there are no edges between $Z_{x}$ and $Y_{y} \cup Z_{y}$. 
Such $i$ exists, because $\left(X_{1}, Y_{1}, Z_{1}\right)$ satisfies these conditions. We claim that $i=R_{1}$.

Suppose that $i<R_{1}$. We choose a connected component $H$ of $G\left[X_{i}\right]$ with chromatic number more than $g_{r}(q) \cdot 2^{R_{1}-i}$ and let $v$ be a vertex in $H$. For $j \geqslant 0$, let $L_{j}$ be the set of all vertices of $H$ whose distance to $v$ is $j$ in $H$. Since $H$ cannot be colored with $g_{r}(q) \cdot 2^{R_{1}-i}$ colors, there exists $t>0$ such that $\chi\left(H\left[L_{t}\right]\right)>g_{r}(q) \cdot 2^{R_{1}-(i+1)} \geqslant g_{r}(q)$. Since $H\left[L_{t}\right]$ has chromatic number at least $g_{r}(q)$, by Theorem [8.1, it contains an induced cycle of length at least $r$. Since $r \geqslant n+3$, by Lemma 6.5, we have $t \geqslant 2$. Let $X_{i+1}:=L_{t}, Y_{i+1}:=L_{t-1}, Z_{i+1}:=L_{0} \cup L_{1} \cup \cdots \cup L_{t-2}$, and let $r_{i+1}$ be the vertex in $L_{0}$. Then $X_{i+1}$ and $Y_{1}, \ldots, Y_{i+1}$ and $Z_{1}, \ldots, Z_{i+1}$ satisfy these conditions, and it contradicts to the choice of $i$. Therefore we have $i=R_{1}$.

Since $\chi\left(G\left[X_{R_{1}}\right]\right)>g_{r}(q), G\left[X_{R_{1}}\right]$ contains an induced cycle $q_{1} q_{2} \cdots q_{m} q_{1}$ with $m \geqslant r$. We apply Proposition 7.4 to the subgraph of $G$ induced on $\left\{q_{1}, q_{2}, \ldots, q_{m}\right\} \cup Y_{1} \cup \cdots \cup Y_{R_{1}}$. Since $m \geqslant r=$ $R_{2} n^{R_{1}}$ vertices, by Proposition 7.4, $G$ contains an $\left(R_{1}, R_{2}\right)$-patched cycle $\left(q_{1} q_{2} \cdots q_{m} q_{1}, S_{1}, \ldots, S_{R_{1}}\right)$ such that for each $j \in\left\{1, \ldots, R_{1}\right\}, S_{j} \subseteq Y_{j}$. Furthermore, since $\omega(G) \leqslant q$, by Proposition 7.3. $G$ contains a simple $(2, M(n))$-patched cycle $\left(q_{1} q_{2} \cdots q_{m} q_{1}, S_{a}^{\prime}, S_{b}^{\prime}\right)$ such that $S_{a}^{\prime} \subseteq S_{a}$ and $S_{b}^{\prime} \subseteq S_{b}$ for some $a$ and $b$ with $1 \leqslant a<b \leqslant R_{1}$. Note that

- there are no edges between $\left\{q_{1}, q_{2}, \ldots, q_{m}\right\}$ and $Z_{a} \cup Z_{b}$,

- there are no edges between $Z_{b}$ and $S_{a}^{\prime}$,

- for each $x \in\{a, b\}$, every vertex of $S_{x}^{\prime}$ has a neighbor in $Z_{x}$,

- for each $x \in\{a, b\}$ and each vertex $v \in N_{G}\left(S_{x}^{\prime}\right) \cap Z_{x}$, there is a path $P$ from $v$ to $r_{x}$ in $G\left[Z_{x}\right]$ with $N_{G}\left(S_{x}^{\prime}\right) \cap V(P)=\{v\}$.

Therefore, by Proposition 7.1, $G$ contains a vertex-minor isomorphic to $W_{n}$, which is contradiction.

\section{References}

[1] A. Bouchet. Isotropic systems. European J. Combin., 8(3):231-244, 1987.

[2] A. Bouchet. Reducing prime graphs and recognizing circle graphs. Combinatorica, 7(3):243$254,1987$.

[3] A. Bouchet. Graphic presentations of isotropic systems. J. Combin. Theory Ser. B, 45(1):58$76,1988$.

[4] A. Bouchet. Connectivity of isotropic systems. In Combinatorial Mathematics: Proceedings of the Third International Conference (New York, 1985), volume 555 of Ann. New York Acad. Sci., pages 81-93, New York, 1989. New York Acad. Sci.

[5] A. Bouchet. $\kappa$-transformations, local complementations and switching. In Cycles and rays (Montreal, PQ, 1987), volume 301 of NATO Adv. Sci. Inst. Ser. C Math. Phys. Sci., pages 41-50. Kluwer Acad. Publ., Dordrecht, 1990.

[6] A. Bouchet. Circle graph obstructions. J. Combin. Theory Ser. B, 60(1):107-144, 1994.

[7] I. Choi, O. Kwon, and S. Oum. Coloring graphs without fan vertex-minors and graphs without cycle pivot-minors. J. Combin. Theory Ser. B, 123:126-147, 2017. 
[8] M. Chudnovsky, A. Scott, and P. Seymour. Induced subgraphs of graphs with large chromatic number. III. Long holes. Combinatorica, 37(6):1057-1072, 2017.

[9] R. Diestel. Graph theory, volume 173 of Graduate Texts in Mathematics. Springer, Heidelberg, fourth edition, 2010.

[10] Z. Dvořák and D. Král'. Classes of graphs with small rank decompositions are $\chi$-bounded. Eur. J. Comb., 33(4):679-683, 2012.

[11] J. F. Geelen. Matchings, matroids and unimodular matrices. PhD thesis, University of Waterloo, 1995.

[12] R. L. Graham, B. L. Rothschild, and J. H. Spencer. Ramsey theory. Wiley-Interscience Series in Discrete Mathematics and Optimization. John Wiley \& Sons, Inc., New York, second edition, 1990. A Wiley-Interscience Publication.

[13] A. Gyárfás. On the chromatic number of multiple interval graphs and overlap graphs. Discrete Math., 55(2):161-166, 1985.

[14] A. Gyárfás. Corrigendum: "On the chromatic number of multiple interval graphs and overlap graphs" [Discrete Math. 55 (1985), no. 2, 161-166; MR0798532 (86k:05052)]. Discrete Math., 62(3):333, 1986.

[15] A. Gyárfás. Problems from the world surrounding perfect graphs. Zastos. Mat., 19:413-441, 1987.

[16] O. Kwon and S. Oum. Unavoidable vertex-minors in large prime graphs. European J. Combin., 41:100-127, 2014.

[17] S. Oum. Rank-width and vertex-minors. J. Combin. Theory Ser. B, 95(1):79-100, 2005.

[18] A. Seidenberg. A simple proof of a theorem of Erdös and Szekeres. J. London Math. Soc., 34:352, 1959.

[19] W. T. Trotter. Combinatorics and partially ordered sets. Johns Hopkins Series in the Mathematical Sciences. Johns Hopkins University Press, Baltimore, MD, 1992. Dimension theory. 